\title{
Localization of sensor nodes in wireless sensor networks using bat optimization algorithm with enhanced exploration and exploitation characteristics
}

\author{
Satinder Singh Mohar ${ }^{1}$ D $\cdot$ Sonia Goyal ${ }^{1} \cdot$ Ranjit Kaur $^{1}$
}

Accepted: 16 January 2022 / Published online: 22 February 2022

(C) The Author(s), under exclusive licence to Springer Science+Business Media, LLC, part of Springer Nature 2022

\begin{abstract}
Wireless sensor networks (WSNs) contain sensor nodes in enormous amount to accumulate the information about the nearby surroundings, and this information is insignificant until the exact position from where data have been collected is revealed. Localization of sensor nodes in WSNs plays a significant role in several applications such as detecting the enemy movement in military applications. The major aim of localization problem is to find the coordinates of all target nodes with the help of anchor nodes. In this paper, two variants of bat optimization algorithm (BOA) are proposed to localize the sensor nodes in a more efficient way and to overcome the drawbacks of original BOA, i.e. being trapped in local optimum solution. The exploration and exploitation features of original BOA are modified in the proposed BOA variants 1 and 2 using improved global and local search strategies. To validate the efficiency of the proposed BOA variants 1 and 2, several simulations have been performed for various numbers of target nodes and anchor nodes, and the results are compared with original BOA and other existing optimization algorithms applied to node localization problem. The proposed BOA variants 1 and 2 outperform the other algorithms in terms of mean localization error, number of localized nodes and computing time. Further, the proposed BOA variants 1 and 2 and original BOA are also compared in terms of various errors and localization efficiency for several values of target and anchor nodes. The simulations results signify that the proposed BOA variant 2 is superior to the proposed BOA variant 1 and existing BOA in terms of several errors. The node localization based on the proposed BOA variant 2 is more effective as it takes less time to perform computations and has less mean localization error than the proposed BOA variant $1, \mathrm{BOA}$ and other existing optimization algorithms.
\end{abstract}

Keywords Node localization · Bat optimization algorithm · Computation time · Target nodes $\cdot$ Localization error

Satinder Singh Mohar

satindersinghmohar66@gmail.com

Extended author information available on the last page of the article 


\section{Introduction}

The modernistic upgradation in wireless technology has inspired the researchers to investigate the various issues faced by wireless sensor networks (WSNs). The enormous sensor nodes are positioned at random or at predefined locations in WSNs in order to gather the data and to transfer the data to other nodes [1-3]. WSNs have been used in various fields such as for monitoring various parameters, such as temperature, pressure and level of air pollutants, for detecting the forest fire and landslides and also used in real-time applications, for example in agriculture and medical areas [4-6]. The localization of sensor nodes plays a significant role in such applications of WSNs. WSN is also used for the management of electronic waste in [7] and in various security applications such as for the protection of secret data from unwanted users, various attacks in VANET and MANET [8-11].

There are various problems faced by WSNs, such as localization, node deployment, routing, energy consumed by sensor nodes, and short lifetime of sensor nodes [12-16]. Enhancing the network lifetime is another issue in WSN which is associated with energy consumed by sensor nodes, and the lifetime of WSNs can be improved by using clustering-based routing protocols [16-18]. Another problem faced by WSN is to provide sufficient coverage by using a few number of sensor nodes [19]. Localization is one of the major problems faced by WSNs, because the data collected by the sensor nodes will be worthless until the location from where the data have been taken is discovered [20]. In WSNs, large number of nodes are placed at random sites whose locations cannot be predetermined. The objective of localization problem is to discover the positions of each sensor node in WSNs [21, 22]. The substitute for the localization problem is that the global positioning system (GPS) can be used to find out the location of sensor nodes. But this approach cannot be used in WSNs because WSNs are comprised of large sensor nodes and using GPS device for each node will increase the cost and complexity of overall network and also consume more power [23, 24].

To overcome these problems, many researchers have introduced several localization techniques. There is no need to use GPS for all sensor nodes, the positions of few nodes are determined through GPS system, and these nodes are known as anchor nodes. With the help of these anchor nodes, the location of unknown nodes termed as target nodes is calculated [25, 26]. The position of anchor node is provided as input to localization process, and another input is determined by measurement techniques [24]. The localization techniques are classified into two types: range-free and range-based localization. The connectivity data are used as another input in range-free localization, and angle of arrival (AOA), received signal strength (RSS), etc., are used as other inputs in range-based localization [27, 28].

To compute the distance between sensor nodes, several techniques such as AOA, RSS, time of arrival (TOA) and time difference of arrival (TDOA) have been used by various researchers [24, 29]. In last few years, various investigators have used many optimization techniques such as particle swarm optimization, bat optimization algorithm, salp swarm algorithm and firefly algorithm for localizing 
the sensor nodes in WSNs. Each optimization algorithm has merits and demerits. These optimization algorithms are based on behaviour and searching ability of various natural systems to determine the food source. The optimization methods can be used to solve the problem of localization of sensor nodes. The concept of optimization is used in every field; for example, deep learning methods are used to diagnose the COVID-19 in [30] and to optimize the genuine rating given by the customer in order to protect from fake reviews in [31].

In this paper, bat optimization algorithm variants are proposed to localize the sensor nodes in the WSNs. The bat optimization algorithm is based on echolocating behaviour of bats. The bat algorithm has various advantages compared to genetic algorithm (GA), particle swarm optimization (PSO), firefly algorithm (FA) and cuckoo search optimization algorithm (CSOA) as it is simple, flexible, converges faster, more accurate, easy to implement and can be used to discover optimal solutions in many applications such as data mining, scheduling tasks in multiple stages, and in designing pressure vessel [32]. Bat optimization algorithm is efficient than other optimization algorithms due to several unique features of BOA which are discussed as below.

\subsection{Frequency tuning}

BOA used echolocation and frequency tuning to find the optimal solutions. The variations in frequency provide the functionality which is similar to main features of PSO and harmony search optimization algorithm (HSOA). Thus, BOA possesses the advantages of PSO and HSOA [32].

\subsection{Automatic zooming}

BOA has unique feature of automatic zooming as compare to GA, PSO, CSOA and FA. Automatic zooming means BOA zooms into the area where optimized solution can be discovered by switching automatically from exploration to exploitation. Due to this feature, BOA has a higher rate of convergence by achieving optimized solution at earlier stage of iterations compared to the above-stated optimization algorithms [32].

\subsection{Parameter control}

PSO and GA have fixed parameters, but parameters of BOA such as loudness, pulse emission rate and iterations can be varied to find optimal solution. This is another advantage of BOA over PSO and GA [32].

The bat optimization algorithm and its variants can be applied to any field of optimization, image processing, classification, data mining, scheduling, feature selection, etc. The BOA can be used to discover optimal solutions for highly nonlinear engineering optimization problems such as car side design and pressure vessel design. BOA can be used to solve multi-stage scheduling tasks, for data compression, for the classification of microarray data, etc. [32]. So BOA is not 
customized for WSN localization; it can be applied to find optimized solutions in various fields stated above.

The performance of node localization based on BOA is better than existing optimization algorithms in terms of localization error and computing time. The bat optimization algorithm is not capable of localizing all the target nodes in the network due to the problem of being stuck in local optimum solution and does not explore in each direction of monitoring area. Therefore, to remove these problems faced by BOA the searching capabilities of original bat optimization algorithm are enhanced by introducing two BOA variants. The major objective of the proposed BOA variants 1 and 2 is to improve the performance of wireless sensor network by localizing all target nodes in the network. In the proposed BOA variant 1 , the exploration characteristic of existing bat optimization algorithm is modified by using improved global searching strategy. In the proposed BOA variant 2 , the improved local searching strategy is used to modify the exploitation characteristic of original BOA.

The novelties of node localization based on the proposed BOA variants are discussed as follows:

1. The searching capabilities of existing BOA are improved by enhancing exploration and exploitation abilities in the proposed BOA variants 1 and 2, respectively.

2. The performance of the proposed BOA variants 1 and 2 is computed and compared in terms of mean localization error, computation time and number of localized nodes with original BOA and other existing optimization algorithms such as particle swarm optimization (PSO) algorithm, grey wolf optimization (GWO) algorithm, butterfly optimization algorithm (BTOA), salp swarm optimization (SSO) algorithm and firefly algorithm (FA).

3. The proposed BOA variants 1 and 2 localized all target nodes and have less mean localization error and computation time than these existing algorithms. The proposed BOA variant 2 is superior to the proposed BOA variant 1 in terms of computing time and mean localization error.

4. In addition to this performance of original BOA, the proposed BOA variants 1 and 2 are also analysed in terms of several types of error, for instance average localization error, normalized localization error and root-mean-square error, and localization efficiency for different scenarios of nodes.

5. The proposed BOA variant 2 is effective than the proposed BOA variant 1 and original BOA for various types of errors.

There are several issues in range-based localization techniques. The performance of AOA localization technique is affected by shadowing and multipath problem which in turn increases the localization error. In order to improve the accuracy of AOA algorithm, large antenna arrays are used, which increases the power consumption and cost of the WSN [33]. In TOA algorithm, synchronization between sender and receiver is required that needs high accuracy clock for synchronization which increases the cost and complexity of the WSN [33]. The accuracy of TDOA algorithm is influenced by humidity and temperature. TDOA 
localization algorithm is least used for low-power WSN [34]. The cost of RSSI localization algorithm is least among AOA, TDOA and TOA localization algorithm because it required less hardware. The multipath propagation, reflection and refraction influence the performance of RSSI algorithm which increases the localization error [34].

Therefore, there are two major issues in range-based localization algorithms, i.e. cost and accuracy. The BOA variants 1 and 2 are proposed to improve the accuracy of localization process in WSN by using improved global and local search strategies. The mean localization error of the proposed BOA variants 1 and 2 is significantly lesser than that of the existing algorithms such as BOA, FA, BTOA, PSO, SSA and GWO. Therefore, the accuracy of WSN is improved by using BOA variants 1 and 2 for localizing the nodes in the WSN. The proposed BOA variants 1 and 2 do not need any additional device which means it does not increase the cost of the WSN. Moreover, both BOA variants 1 and 2 converge faster and have less computation time than the existing algorithms.

The remaining part of the paper is organized as follows: Sect. 2 provides the review of various optimization techniques used for node localization problem. In Sect. 3, the bat optimization algorithm is discussed in detail. The proposed BOA variants 1 and 2 are explained in Sect. 4. Node localization in WSNs using the proposed BOA variants 1 and 2 is described in Sect. 5. Section 6 represents the simulation results and discussion, and Sect. 7 provides the conclusion.

\section{Literature review}

Several researchers have applied various optimization algorithms to solve the problem of localization of nodes in WSNs. Particle swarm optimization (PSO) was suggested in [35] to localize the nodes in WSNs as well as to reduce average localization error. Iterative localization methods such as PSO and bacterial foraging algorithm (BFA) were recommended in [36] to solve the multi-objective localization problem. The suggested algorithms had reduced the power consumed by nodes and also taken less time to define the coordinates of target nodes in WSNs. Bees optimization algorithm was used in [37] to decrease the average error of target nodes from anchor nodes. Two cases were considered for positioning the anchor nodes in deployment area. In the first method, each target node is surrounded by more than three anchor nodes and beacon nodes were deployed in the centre of monitoring region in the second method [37].

To localize the nodes in three deployment (3D) areas, stochastic PSO was suggested in [38]. The stochastic PSO algorithm had positioned the target nodes more accurately than other methods based on PSO. To enhance the localization precision and convergence rate, hybrid bio-inspired optimization technique based on PSO and BFO was used in [39]. The PSO and BFO algorithm had localized more number of sensor nodes and decreased the energy consumed by nodes than other algorithms. To minimize the deployment cost by using sufficient amount of anchor nodes for localizing every target node in WSNs, novel genetic optimization technique was suggested in [40]. To attain convergence at less iteration and to define the coordinates of target 
nodes more accurately, cuckoo search optimization algorithm (CSOA) was recommended in [41]. The CSOA was superior to other optimization algorithms in discovering the overall optimal outcomes. To remove the flip uncertainty issue in WSNs, the gravitational search optimization algorithm was modified in [42]. The results had shown that recommended algorithm also reduced the average localization error.

To enhance the lifetime of WSNs and to minimize the computation time, binary PSO was used in [43] to localize the target nodes in the network. RSS had been used to compute the distance of target nodes from anchor nodes and also saved energy of sensor nodes. A multi-objective two-phase PSO algorithm was developed in [44] to improve the performance of WSNs and to eliminate flip uncertainty issue. The twophase PSO algorithm had taken less time to localize all the target nodes in WSNs. Modified bat algorithm was suggested in [45] to determine the optimum value of error and to enhance the accuracy of localization process. The computation time of suggested algorithm was less and localized all target nodes in the network. To reduce the computing time and to find the overall optimal solutions, parallel firefly algorithm was developed in [46] which used RSS for localization of sensor nodes. To reduce the approximation error and to improve the precision of localization, improved DVHop method was used in [47]. The modified DV-Hop algorithm decreased the localization error as compared to other localization methods.

To resolve the localization issue in WSNs and to localize more sensor nodes, flower pollination (FP) algorithm was used in [48]. The FP algorithm was more accurate than other PSO methods. To localize mobile nodes, orthogonal teaching-learning-based optimization (OTLBO) approach was suggested in [49]. OTLBO was more complex but enhanced network life and coverage. To localize the nodes in $3 \mathrm{D}$ area, range-free firefly algorithm was introduced in [50]. To reduce the computation complications, fuzzy logic was used to determine the nonlinearity between distance and RSS. The firefly algorithm discovered coordinates of all sensor nodes in less time than other algorithms. To discover the positions of target nodes more precisely, artificial bee colony algorithm (ABC) was used in [51]. ABC localized the sensor nodes more accurately but taken more time for computation as compared to other techniques. The localization error function was amended in [20] to increase the accuracy, and only two anchor nodes were considered in the range of target node. To obtain greater convergence rate, chicken swarm optimization (CSO) was used in [52] to discover the positions of target nodes. The accuracy of CSO algorithm was more than PSO algorithm. To reduce the computing time, grey wolf optimization (GWO) algorithm was suggested in [53] and GWO localized more target nodes than other algorithms.

Butterfly optimization algorithm (BTOA) was introduced in [54] to improve the performance of WSNs by localizing target nodes with more accuracy. To show the effectiveness of BTOA, dimensions of deployment area were varied and effected by various noises and the results revealed that the BTOA was superior in terms of accuracy and computation time. The novel algorithm based on PSO for locating random mobile nodes in WSNs was suggested in [55]. Firstly, RSS was used to compute the distance of anchor nodes from target nodes and then few anchor nodes were positioned in monitoring region with some angles. The PSO had less mean convergence time than other algorithms. 
Monarch BTOA (MBTOA) was applied to multi-stage localization issue in WSNs in [56], and the performance of MBTOA was compared with other optimization algorithms. The results signified that MBTOA had better performance than other algorithms in terms of average localization error. Elephant herding optimization (EHO) algorithm was introduced in [57] to decrease the mean square error in localization problem and to enhance the performance of WSNs by localizing random target nodes in the network. To improve the lifetime of large WSNs, multiobjective whale optimization algorithm (MO-WOA) was used in [58]. MO-WOA had reduced the number of anchor nodes and also decreased the energy consumed by network. Hybrid PSO with neighbourhood search was suggested in [59] for localization of nodes in outdoor monitoring area. The suggested algorithm had less computation time and localization error than that of various algorithms based on PSO. To increase the accuracy of node localization, salp swarm optimization (SSO) algorithm was applied in [60]. SSO algorithm had taken less time for computation and localized more target nodes.

Three variants of naked mole-rat (NMR) algorithm were presented in [61] to enhance the searching abilities of NMR algorithm and to localize the nodes in two-dimensional and three-dimensional WSNs. The results revealed that the NMR algorithms had faster convergence speed and small localization error than other algorithms. The genetic algorithm (GA) was recommended in [62] for DV-Hop localization for determining the position of newly deployed sensor nodes in three different topologies: random, C-shape, and W-shape topology. The genetic algorithm had less computation time and localization error than other existing algorithms. To reduce the localization error, artificial bee colony (ABC) algorithm was used in [63] for unmanned aerial vehicles anchor nodes. The simulation results have shown that ABC algorithm had 25\% more accuracy than the existing algorithms. Dragonfly-firefly algorithm (DFA) was suggested in [64] to discover global optimal solutions for localization problem. The DFA algorithm had high convergence speed and less localization error. The genetic algorithm was hybrid with PSO algorithm in [65] for localization for nodes in three-dimensional indoor WSN.

Improved CSOA was introduced in [66] to improve the localizing accuracy of sensor nodes in WSNs. The localization error of improved CSOA was less than that of CSOA. To locate the sensor nodes accurately in 3D environment, the teaching-learning-based optimization (TLBO) algorithm was improved with learning enthusiasm in [67]. The suggested algorithm provided equal balance between exploitation and exploration characteristics and also reduced localization error for 3D deployment area. DV-Hop algorithm was modified in [68] with class topper optimization algorithm to discover the positions of target nodes in WSNs. The accuracy was improved, and localization error was reduced in suggested algorithm. Further, to improve the accuracy and robustness, the DV-Hop algorithm was improved by using squirrel search algorithm in [69] to localize target nodes in WSNs. The recommended algorithm in [65] had fast convergence rate, high accuracy and more robustness.

Bird swarm optimization was hybridized with quasi-affine evolutionary algorithm in [70] to localize the nodes with more accuracy in WSN. RSS was used to discover the location of sensor nodes, and the bird swarm optimization had more node 
connectivity and less localization error. For underwater WSN concept of Doppler shift was used along with genetic algorithm in [71] to discover the coordinates of target nodes. The Doppler shift discovered the position of all target nodes, and genetic algorithm had minimized the localization error. The convergence rate and accuracy of suggested GA were more than the existing algorithms. Improved self-adaptive PSO algorithm was suggested in [72] to improve the accuracy and to reduce the power consumed by sensor nodes under various scenarios of error and sensor node values.

The DV-Hop localization was enhanced by using runner root optimization algorithm for node localization in [73] to overcome demerits of original DV-Hop algorithm. The computing time and localization error of enhanced DV-Hop algorithm were less compared to other algorithms. To locate the nodes in three-dimensional WSN, adaptive FP algorithm was introduced in [74]. The simulation results had shown that adaptive Fp algorithm was superior to other algorithms in terms of convergence speed and localization error. The PSO algorithm was used for DV-Hop localization of displaced sensor nodes in [75], and radio irregularity model was considered for simulation. The elapsed time, localization error and energy consumption of PSO algorithm were less than those of the existing algorithms. The fruit fly optimization algorithm was used in [76] to locate target nodes in the WSN. The fruit fly optimization algorithm had more accuracy and faster convergence speed compared to other algorithms. To detect high-degree nodes, distance model was used to implement resource graph in [77]. The Hessian regularization technique in iterative manner was used in [78] for localization of sensor nodes. The Hessian regularization algorithm had $70 \%$ more accuracy than the existing algorithms.

In last few years, various researchers had used several optimization techniques to solve the localization problem in the WSN. The advantage of optimization algorithms used for localization of sensor nodes is that the localization error and computation time reduced to significant amount, but there is still possibility to further reduce the computing time and localization error by using efficient evolutionary algorithms. There are several issues in localization, such as scalability, cost, convergence time and accuracy. Scalability defines the capability to enlarge the WSN by increasing the nodes in the WSN, and it is an important factor to estimate the performance of WSN. Another issue in localization is the cost of the network. The major goal of the researchers is to propose the cost-effective optimization algorithm for localization, but there is a trade-off between the accuracy and cost of WSN. Anchor-to-target nodes ratio is another factor which affects the cost of the WSN by increasing the number of anchor nodes; the localization error decreases, which increases the accuracy of WSN. But it also increases the cost of WSN because GPS is required to determine the position of anchor nodes.

The convergence time signifies the time taken by the optimization algorithm to localize all target nodes in the WSN. The foremost aim of optimization algorithm used for localization is to discover the position of target nodes accurately. The accuracy of optimization algorithm for localization is represented as average localization error. If the value of average localization error is less, then optimization algorithm is more accurate.

These issues motivate the researchers to suggest novel optimization algorithms to localize the sensor nodes in more accurate way and in less time. To locate the sensor 
nodes more accurately in less time, the searching abilities are enhanced in the proposed BOA variants 1 and 2 by using improved global and local search strategies, respectively. Both the proposed BOA variants 1 and 2 are more accurate because the mean localization error and average localization error of BOA variants 1 and 2 are less than those of BOA and various existing optimization algorithms such as FA, PSO, GWO, SSA and BTOA. The proposed BOA variants 1 and 2 converge faster at 25 iterations due to enhanced searching abilities, whereas other existing optimization algorithms mentioned above converge at 100 iterations. The proposed BOA variants 1 and 2 localized all target nodes in the WSN for 150 target nodes by using only 20 anchor nodes, whereas the above-stated optimization algorithms utilized 35 anchor nodes. Therefore, the cost of the proposed BOA variants 1 and 2 is less compared to the existing algorithms because the proposed BOA variants 1 and 2 are using only 20 anchor nodes to localize 150 target nodes. The localization error of the proposed BOA variant 2 is smaller than all existing algorithms, BOA and proposed BOA variant 1 for 150 target nodes and 20 anchor nodes.

\section{Bat optimization algorithm}

Bat optimization algorithm (BOA) is suggested by Yang to discover the global optimal solutions [79]. BOA is a population-based optimization technique and is enthused by enthralling actions of bat group such as determining the location of food source and categorizing numerous types of insects in entire dark atmosphere. The innovative echolocation skill of bat algorithm motivates the investigators to analyse the bat algorithm. Sonar designated as echolocation is used by entire bat group to find the food source location and to evade hindrances. The bat group can find the location of food source by sending low- and high-frequency sound pulses, and these pulses strike and return to the bats $[15,79]$.

The echolocation aspects of bat algorithm are faultless in agenda of the following recommendations $[15,79]$ :

- Echolocation is used by each bat to calculate the distance, and bats have the ability to discriminate between food source and contextual hindrances.

- In BOA, each bat is assigned some value of velocity $V$, frequency $F$, initial position $X$ and loudness parameter $A$ to discover the food source. Bats can amend the frequencies of released pulses, as well as they have the ability to alter the pulse emission rate $r$ whose value lies between 0 and 1 depending upon the nearness of the food source.

- Moreover, the value of loudness can be varied in various ways, but in many circumstances loudness is altered from the largest value $A_{\max }$ to the smallest value $A_{\text {min }}$.

At preliminary phase, the bats are assigned initial velocities and placed at random sites in the investigation area. The velocities $V_{i}^{T}$, frequencies $F_{i}$ and positions $X_{i}^{T}$ of bats are updated at time $T$ and are stated by the following equations: 


$$
\begin{gathered}
F_{i}=F_{\min }+\left(F_{\max }-F_{\min }\right) \alpha \\
V_{i}^{T}=V_{i}^{T-1}+\left(X_{i}^{T}-X^{\$}\right) F_{i} \\
X_{i}^{T}=X_{i}^{T-1}+V_{i}^{T}
\end{gathered}
$$

where $V_{i}^{T}, F_{i}$ and $X_{i}^{T}$ designate the velocity, frequency and location of $i$ th bat at time $T$, respectively. The highest and lowest frequencies of bat algorithm are signified as $F_{\min }$ and $F_{\max } . \alpha$ represents the random variable, and its value corresponds to [0,1] interval. $X^{\$}$ denotes the global optimal solution found by relating locations of every bat at all iterations [79]. Predominantly, every bat is assigned random frequency whose value is chosen from $\left[F_{\min }, F_{\max }\right]$. If new solutions for bats are accepted, then the values of loudness and pulse emission rate are updated by using the following equations:

$$
\begin{gathered}
A_{i}^{T+1}=\beta A_{i}^{T} \\
r_{i}^{T+1}=r_{i}^{0}\left[1-e^{-\gamma T}\right]
\end{gathered}
$$

where $\beta, \gamma$ are constants and $A_{i}^{T}, r_{i}^{0}$ signify the initial value of loudness and pulse emission rate, respectively. Table 1 shows the pseudocode for BOA.

Table 1 Pseudocode for bat optimization algorithm

\section{Begin}

State objective function $f(X)$, where $X=\left(X_{1}, X_{2}, X_{3}, \ldots \ldots \ldots \ldots X_{d}\right)^{T}$

Define bat group size, initial positions $X_{i}$ and velocities $V_{i}$ where $i=(1,2,3, \ldots \ldots . n)$

Initialize frequencies of bats $F_{i}$ at $X_{i}$

Set the value of loudness $A_{i}$ and pulse emission rate $r_{i}$

While $(T<$ Maximum iterations $)$

Create new solutions by altering the frequency, velocity and position of bats by using equations 1,2 and 3

If $\left(r\right.$ and $\left.>r_{i}\right)$

Discover the best value of solution by equating all solutions and store its value

Local solution is generated about the selected best values

End if

Bats are flying arbitrarily and new solutions are generated

If $\left(\right.$ rand $\left.<A_{i}\right) \&\left(f\left(X_{i}\right)<f\left(X^{\$}\right)\right)$

New solutions are accepted

Increase the value of $r_{i}$ and decrease $A_{i}$ by using equations 4 and 5

End if

End while

Select the bat which has best solution

End

Post processing of results and visualization 


\section{Proposed bat optimization algorithm variants}

The original bat optimization algorithm localized sufficient amount of sensor nodes. The mean localization error and computation time of bat optimization algorithm are less compared to other existing algorithms, but success rate of BOA is less as it does not localize all the target nodes in the network. BOA is not capable of exploring every direction in the search area. So it is necessary to modify the bat optimization algorithm in order to overcome these issues and to further reduce the mean localization error and computation time. In this paper, two variants of bat optimization algorithm are proposed. The bat optimization algorithm variants are introduced to provide efficient performance of WSNs by enhancing the exploration and exploitation characteristics of bat algorithm. The exploration feature of bat optimization algorithm is enhanced by introducing improved global search strategy in BOA variant 1 . The exploitation characteristic of BOA is enhanced by using improved local search strategy in BOA variant 2 . These variants are discussed in detail as follows:

\subsection{BOA variant 1 based on improved global search strategy}

When bat optimization algorithm stuck in local optimum solution, it is impossible to obtain global optimal solution. So it is necessary to direct the existing solution towards the overall optimal solution. The improved global search strategy is used to enhance the global search abilities of BOA in the proposed BOA variant 1 . The main logic behind improved global search strategy is to find the global optimum solution with the help of different procedures and by exploring in larger region of the search area.

$N$ number of target nodes and $M$ number of anchor nodes are randomly deployed in the search area, and population size is $P$ which means that there will be $P$ number of candidate solutions. In the proposed BOA variant 1 , two frequencies are generated for updating the velocity of bats and these frequencies are denoted as $F_{i}(1)$ and $F_{i}(2)$ for $i$ th sensor nodes. The values of these frequencies are computed by using Eqs. 6 and 7:

$$
\begin{aligned}
& F_{i}(1)=F_{\text {min }}+\left(F_{\text {max }}-F_{\text {min }}\right) \delta \\
& F_{i}(2)=F_{\text {min }}+\left(F_{\text {max }}-F_{\text {min }}\right) \varepsilon
\end{aligned}
$$

The value of $F_{i}(1)$ and $F_{i}(2)$ depends upon $\delta, \varepsilon, F_{\min }$ and $F_{\max } . \delta$ and $\varepsilon$ are random numbers whose values lie between 0 and 1 . Then, velocity and position of bats are updated by using the following equations:

$$
\begin{gathered}
V_{i}^{T}=V_{i}^{T-1}+\left(X^{b}-X_{i}^{T}\right) * F_{i}(1)+\left(X^{w}-X_{i}^{T}\right) * F_{i}(2) \\
X_{i}^{T}=X_{i}^{T-1}+V_{i}^{T}
\end{gathered}
$$


where $X^{b}$ and $X^{w}$ signify the best and worst solutions and $X_{i}^{T}$ represents the current solution of $i$ th bat at time $T$. In original bat optimization algorithm, new solutions are generated around the best values and gradually BOA trapped in local optimum solution. In original bat algorithm, the bats are moving only around the best solution and do not explore the entire region. In the proposed BOA variant 1, the search space for bats is increased in order to discover global optimum value. The bats in the proposed BOA variant 1 will explore the whole search space from the best solutions to the worst solution and find overall optimal solution. If optimization problem is of minimization, the values of $X^{b}$ and $X^{w}$ are determined as follows:

$$
\begin{aligned}
X^{b} & =\min (f(X)) \\
X^{w} & =\max (f(X))
\end{aligned}
$$

Where $f(X)$ is the objective function of optimization problem. After updating the positions of all bats, the objective function is computed for every bat and then again the values of worst and best solutions are calculated at the end of the first iteration. These best and worst values are used in updating the velocities of bats in the next iteration and so on.

\subsection{BOA variant 2 based on improved local search strategy}

The proposed BOA variant 1 localized all the target nodes in the wireless sensor network and has smallest mean localization error than other existing algorithms applied to node localization problem. The proposed BOA variant 1 takes less time to localize all the nodes and converges faster compared to other algorithms. But there is a tendency to include the worst solution while updating the velocity of bats. To overcome this problem and to further decrease the mean localization error and computation time, BOA variant 2 is suggested. Improved local search strategy is used to improve the local search capabilities of BOA in the proposed BOA variant 2. The foremost logic of improved local strategy is to use the local data available and current best solution to generate the new solutions for bats.

The improved local strategy used current best solution discovered and worst solution to update the velocity of bats. The proposed BOA variant 2 searches in small area which is nearer to the best solution discovered till now, and it does not include the worst solution. The velocity of bats is modified in the proposed BOA variant 2 using the following equation:

$$
V_{i}^{T}=V_{i}^{T-1}+\left(X^{b}-X_{i}^{T}\right) * F_{i}(1)-\left(X^{w}-X_{i}^{T}\right) * F_{i}(2)
$$

In this equation, the first term $\left(X^{b}-X_{i}^{T}\right) * F_{i}(1)$ specifies that the movement of the solution is towards the best solution which means that the new solution which is generated is closer to the best value. The second term $-\left(X^{w}-X_{i}^{T}\right) * F_{i}(2)$ represents that the solution is avoiding the worst value. The values of $F_{i}(1)$ and $F_{i}(2)$ are computed using Eqs. 6 and 7. The improved local search strategy exploits the small area around the best solution, and it does not explore the entire search space. 
After modifying the velocity of each bat, the locations of bats are updated using Eq. 9. The proposed BOA variant 2 always travels nearer to the local best solution at each iteration and try to move away from the worst value.

There are four major issues in node localization problem, which are convergence rate, mean localization error, computation time and number of localized nodes. The mean localization error and computation time of the proposed BOA variants 1 and 2 are less than those of BOA and existing algorithms such as FA, BTOA, PSO, GWO and SSA. Both proposed BOA variants 1 and 2 localized all target nodes and converge at 25 iterations, while the above-stated existing algorithms and BOA localized all target nodes and converge at 100 iterations.

\section{Node localization using the proposed BOA variants 1 and 2}

The coordinates of target nodes are estimated by using distributed single hop range-based approach. The positions of target nodes are computed with the help of few anchor nodes, and these nodes know their location with the help of GPS. The major goal of localization problem is to discover the location of target nodes and to minimize the objective function. Table 2 shows the list of abbreviations used in the localization process.

Table 2 List of abbreviations

\begin{tabular}{ll}
\hline Symbols & Name of abbreviations \\
\hline$N$ & Target nodes \\
$M$ & Anchor nodes \\
$M I$ & Maximum iterations \\
$R$ & Transmission range of nodes \\
$P$ & Population size \\
$A$ & Loudness \\
$r$ & Pulse emission rate \\
$V$ & Velocity \\
$F_{\text {max }}$ & Maximum frequency \\
$F_{\min }$ & Minimum frequency \\
$N_{L}$ & Total number of localized nodes \\
$M S E$ & Mean square error \\
$r a n d$ & Random number whose value \\
& lies between 0 and 1 \\
$d_{\min }$ & Minimum distance \\
$A L E$ & Average localization error \\
$M L E$ & Mean localization error \\
$N L E$ & Normalized localization error \\
$R M S E$ & Root-mean-square error \\
$L E$ & Localization efficiency \\
\hline &
\end{tabular}


The flow chart for node localization based on the proposed BOA variants is shown in Fig. 1. The various steps which are used to discover the coordinates of $N$ target nodes using the proposed BOA variants are discussed as follows:

Step 1 Firstly, $N$ target nodes and $M$ anchor nodes are deployed arbitrarily in the monitoring area. The anchor nodes know their position through GPS device. The target nodes and anchor nodes have transmission range $R$ and also describe the values of various parameters such as $A, r, V, M I, R, P, F_{\max }$ and $F_{\min }$. The stopping criteria should be defined, i.e. minimum value of error.

Step 2 The distance of every target node is computed from all anchor nodes. Let us suppose that $(x, y)$ is the coordinates of target node which is to be discovered and $\left(x_{i}, y_{i}\right)$ is the coordinates of $i$ th anchor node. The distance $\left(d_{i}\right)$ between target node and $i$ th anchor node is calculated as follows:

$$
d_{i}=\sqrt{\left(x-x_{i}\right)^{2}+\left(y-y_{i}\right)^{2}}
$$

The distance $\left(d_{i}\right)$ is changed by Gaussian noise $\left(n_{i}\right)$ as shown in Eq. 14. The value of $n_{i}$ which affects the computed distance lies in the range $d_{i} \pm\left[\frac{P_{n}}{100}\right]$ where $P_{n}$ represents the percentage of Gaussian noise in the computed distance.

$$
\widehat{d}_{i}=d_{i}+n_{i}
$$

The range-based localization algorithms which compute the distance between two nodes are susceptible to Gaussian noise [80]. The major sources of Gaussian noise in WSN arise during acquisition process, for example sensor noise produced by high temperature, weak illumination and transmission such as electronic circuit noise.

The Gaussian noise is entirely characterized by mean and variance, and it is easy to compute the mean and variance if noise is Gaussian. This is the main benefit of using Gaussian noise because computing the variance and mean is easy than the computation of higher-order parameters that are required when non-Gaussian noise is used [80].

The effect of noises on localization process was analysed in [81] in which noisy disk model was considered. Noisy disk model had two components: connectivity and noise. The noise component specified the error distribution between the computed distances from the actual distances and noise may be Gaussian or uniform. The another component, i.e. connectivity, signified the maximum distance between two sensor nodes at which estimated distance can be computed. It was observed that non-Gaussian noise had increased the localization error by a factor of 4.5 and Gaussian noise had increased the error by a factor of 2.5. Thus, Gaussian noise has less localization error than non-Gaussian noise [81].

Noise is random in nature, and to compute the probability of events a mathematical model is used. Gaussian distribution has zero mean and shown as a bellshaped curve which is symmetrical about mean value. The distance between the sensor nodes is affected by random noise. Adding the Gaussian noise to distance means to create the sequence of random numbers. In this paper, target nodes and sensor nodes are randomly placed into the monitoring area. Therefore, Gaussian 


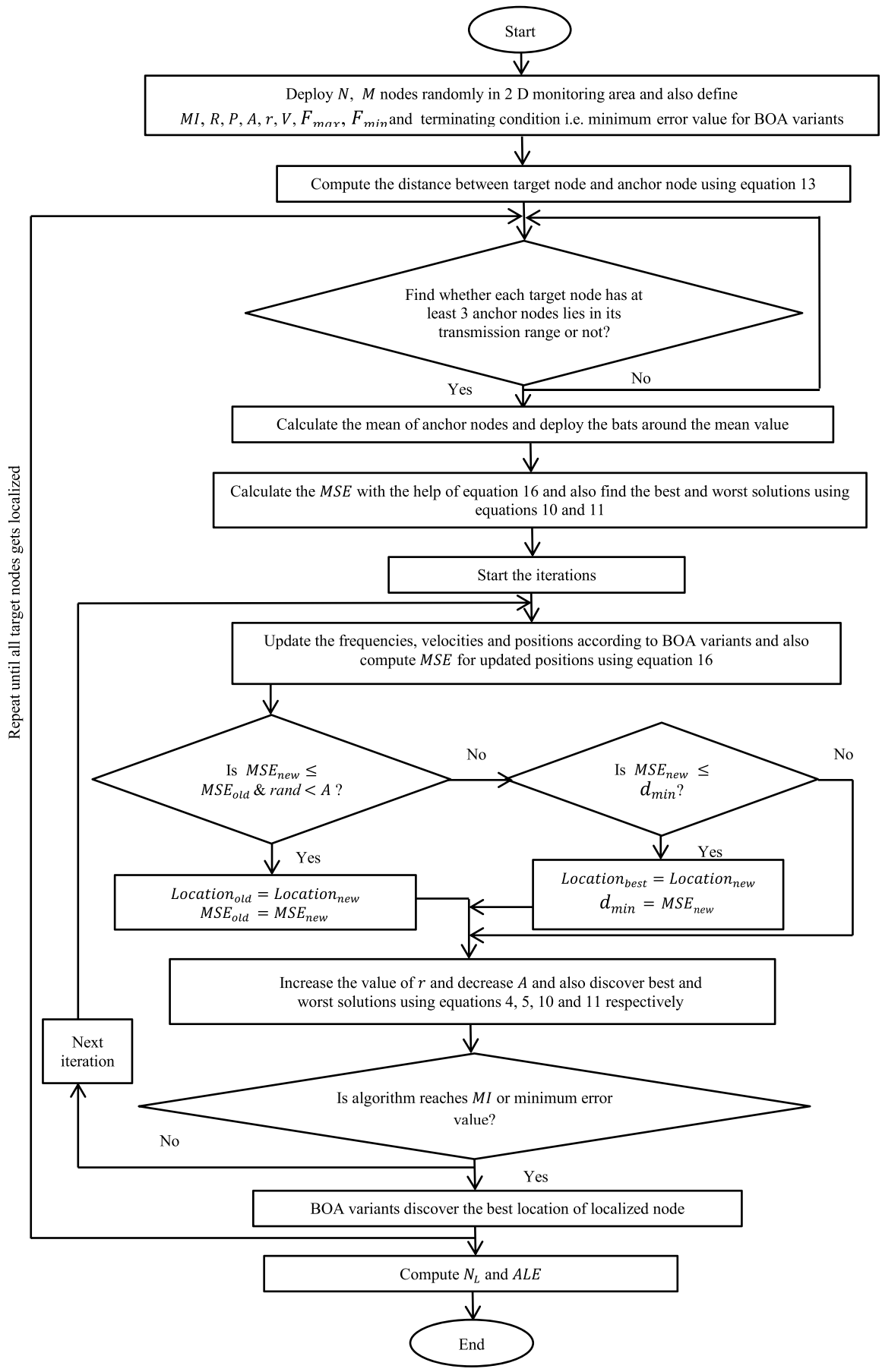

Fig. 1 Flow chart for node localization based on BOA variants in WSNs 
noise is added in distance to introduce average number of errors in the computed distance.

Step 3 The target node is assumed to be localizable node if that target node has at least three anchor nodes that lie in its transmission range. Determine whether each target node has at least three anchor nodes in its range or not. The distances calculated from three or more anchor nodes are used to minimize the localization error between the estimated distance and actual distance.

Step 4 For every target node which is to be localizable each optimization algorithm runs individually to discover the coordinates of target node. Initially, bats are deployed by calculating the mean of anchor nodes which are lying in the transmission range of localizable node as follows:

$$
\left(x_{m}, y_{m}\right)=\left(\frac{1}{M} \sum_{i=1}^{M} x_{i}, \frac{1}{M} \sum_{i=1}^{M} y_{i}\right)
$$

where $M$ is the number of anchor nodes that lies in the transmission range of localizable target node.

Step 5 Each optimization algorithm discovers the coordinates of target node and also reduces the localization error. The mean square error between anchor node and target node is the objective function of node localization problem. The mean square error $(M S E)$ is reduced by using efficient optimization algorithm and can be described as follows:

$$
\text { Objective function }=M S E=f(x, y)=\frac{1}{M}\left(\sum_{i=1}^{M} \sqrt{\left(x-x_{i}\right)^{2}+\left(y-y_{i}\right)^{2}}-\hat{d}_{i}\right)^{2}
$$

where $M$ signifies the number of anchor nodes which lies in the transmission range of target node and its value should be always greater than 3. Also, determine the values of best and worst solutions with the help of Eqs. 10 and 11. Initialize the iterations for BOA variants.

Step 6 Update the values of frequency, velocity and position of bats according to BOA variants. Then, compute MSE for updated positions of bats.

Step 7 Check that new value of MSE is less than or equal to old value of $M S E$ and value of random number ( $r a n d)$ is less than loudness parameter $(A)$. If yes, then store new location of bat in old location and new MSE value in previous MSE value and move to step 9, otherwise go to step 8 .

Step 8 Determine whether new value of MSE is less than or equal to minimum distance value $\left(d_{\text {min }}\right)$. If yes, then select temporary location of bat as new best location and new $M S E$ value as $d_{\min }$, otherwise go to step 9. 
Step 9 Increase the value of pulse emission rate $(r)$ and decrease the value of loudness parameter $(A)$ with the help of Eqs. 4 and 5. Also, find the best and worst solutions using Eqs. 10 and 11.

Step 10 The optimum value of $(x, y)$ is discovered by optimization algorithm after running the algorithm for number of iterations and by reducing the value of objective function. Find that algorithm has reached the value of maximum iterations or minimum error is obtained. If yes, then move to step 11, otherwise go to next iteration and repeat the steps from 6 to 10 .

Step 11 Check whether all target nodes are localized by using the proposed BOA variants. If yes, then go to step 12, otherwise repeat the steps from 3 to 11 until every node is localized in the monitoring area.

Step 12 After finding the coordinates of all target nodes, the total localization error is calculated. It is computed by taking the average of square root of distance of localized nodes coordinates $\left(X_{i}, Y_{i}\right)$ from the real node coordinates $\left(x_{i}, y_{i}\right)$ and described as follows:

$$
\text { Average localization error }(A L E)=\frac{1}{N_{L}} \sum_{i=1}^{N} \sqrt{\left(X_{i}-x_{i}\right)^{2}+\left(Y_{i}-y_{i}\right)^{2}}
$$

where $N_{L}$ is the total number of localized nodes.

The performance of node localization using optimization algorithm is influenced by average localization error $(A L E)$ and number of unlocalized nodes $\left(N_{N_{L}}\right)$ which is calculated by using Eq. 18. The optimization algorithm used for node localization will be effective if it has smaller $A L E$ and $N_{N_{L}}$.

$$
N_{N_{L}}=N-N_{L}
$$

\section{Simulation results and discussion}

To verify the performance of the proposed BOA variants 1 and 2, the simulations are executed on MATLAB R2016a software using laptop having Intel Core i3 processor, $64 \mathrm{~GB}$ memory and $2.40 \mathrm{GHz}$ CPU. The anchor nodes and target nodes are

Table 3 Simulation parameters for WSNs

\begin{tabular}{ll}
\hline Parameters & Values \\
\hline Monitoring area & $100 \mathrm{~m} \times 100 \mathrm{~m}$ \\
Target nodes, $N$ & Varied from 25 to \\
& 150 \\
Anchor nodes, $M$ & Varied from 10 to 35 \\
Transmission range of nodes, $R$ & $30 \mathrm{~m}$ \\
Maximum iterations, $M I$ & 100 \\
Ranging error & $2 \%$ \\
\hline
\end{tabular}


arbitrarily deployed in the monitoring area of $100 \mathrm{~m} \times 100 \mathrm{~m}$, and the transmission range of all sensor nodes is set to $30 \mathrm{~m}$. To check the effectiveness of the proposed BOA variants 1 and 2, their performances are compared with original bat optimization algorithm (BOA). The simulation parameters for localization of nodes in WSNs are presented in Table 3.

\subsection{Parameters set-up}

The parameters of existing BOA and the proposed BOA variants 1 and 2 are shown in Table 4. To perform the comparison of the proposed BOA variants 1 and 2 with existing BOA, the objective function of each algorithm is evaluated $P \times M I$ times except optimal value is obtained earlier, where $P$ is the population size and $M I$ is the maximum iterations. The population size is kept 20 for each algorithm. Moreover, each algorithm has some extra parameters that control the performance of algorithms. $F_{\max }$ and $F_{\min }$ of existing BOA and the proposed BOA variants 1 and 2 have been fixed to $0.05 \mathrm{kHz}$ and $0.01 \mathrm{kHz}$, respectively. The loudness and pulse emission rate for each algorithm are set as 0.2 and 0.5 initially.

\subsection{Evaluation metrics}

The performance of the proposed BOA variants 1 and 2 is evaluated in terms of mean localization error $(M L E)$, average localization error $(A L E)$, computation time $T(s)$, normalized localization error $(N L E)$, root-mean-square error $(R M S E)$ and localization efficiency $(L E)$. The $A L E$, computation time, MLE, RMSE, NLE and $L E$ are explained as follows:

Average Localization Error (ALE) The accuracy of optimization algorithm used for localizing target nodes can be calculated by finding the error between the estimated coordinates and actual coordinates of target nodes, summing the error for all target nodes and then taking the average of total error. The average localization error signifies the accuracy of optimization algorithms used for localization. The average localization error $(A L E)$ is already explained in Eq. 17.

Computation Time $T(s)$ The computation time $T(s)$ is defined as total time taken by the optimization algorithm for performing all the computations during localization of all target nodes in WSN. The computation time $T(s)$ is computed with the help of tic toc function.

Table 4 Parameters of algorithms

\begin{tabular}{|c|c|}
\hline Parameters & Values \\
\hline Population size, $P$ & 20 \\
\hline Maximum frequency, $F_{\max }$ & $0.05 \mathrm{kHz}$ \\
\hline Minimum frequency, $F_{\min }$ & $0.01 \mathrm{kHz}$ \\
\hline Loudness, $A$ & 0.2 \\
\hline Pulse emission rate, $r$ & 0.5 \\
\hline
\end{tabular}


Mean Localization Error ( $M L E)$ The average localization error ( $A L E)$ is normalized in terms of units of transmission range of sensor nodes known as mean localization error $(M L E)$. $M L E$ is also known as average localization error per meter and computed by using the following equation:

$$
\text { Mean localization error }(M L E)=\frac{1}{N_{L}} \frac{\sum_{i=1}^{N} \sqrt{\left(X_{i}-x_{i}\right)^{2}+\left(Y_{i}-y_{i}\right)^{2}}}{R}
$$

Normalized Localization error (NLE) and Root-Mean-Square Error (RMSE) The performance of BOA and the proposed BOA variant 1 and variant 2 is compared in terms of normalized localization error $(N L E)$ which is defined as the ratio of standard deviation to the transmission range of sensor node and root-mean-square error (RMSE) which are described by the following equations:

$$
\begin{gathered}
\text { Normalized localization error, } N L E(\%)=\frac{1}{R} \sqrt{\frac{1}{N_{L}} \sum_{i=1}^{N} \sqrt{\left(X_{i}-x_{i}\right)^{2}+\left(Y_{i}-y_{i}\right)^{2}}} \times 100 \% \\
\text { Root - mean - square error }(R M S E)=\sqrt{\frac{1}{N_{L}} \sum_{i=1}^{N} \sqrt{\left(X_{i}-x_{i}\right)^{2}+\left(Y_{i}-y_{i}\right)^{2}}}
\end{gathered}
$$

Localization Efficiency ( $L E)$ The performance of BOA and the proposed BOA variants 1 and 2 is also compared in terms of localization efficiency $(L E)$ which is defined as a ratio of total number of localized nodes $\left(N_{L}\right)$ to the number of target nodes $(N)$ and is described as follows:

$$
\text { Localization efficiency, } \operatorname{LE}(\%)=\frac{N_{L}}{N} \times 100 \%
$$

\subsection{Comparison of various optimization algorithms used for node localization}

The proposed BOA variants 1 and 2 are compared with the existing BOA. The initial deployment of target nodes and anchor nodes is arbitrary in original BOA and the proposed BOA variants 1 and 2 . Therefore, in order to reduce the influence of randomness each algorithm of BOA and proposed BOA variants 1 and 2 is run for 50 times and the best value of computation time, number of localized nodes and different errors are selected. The localization of sensor nodes based on original BOA and the proposed BOA variants 1 and 2 is shown in Figs. 2, 3 and 4, respectively.

From the figures, it is obvious that the success rate of both the proposed BOA variants 1 and 2 is high because both the proposed BOA variants 1 and 2 localized more target nodes in WSNs than original BOA. The node localization based on the proposed BOA variant 2 is more stable because it has less value of mean localization error than that of the proposed BOA variant 1 and existing BOA. 


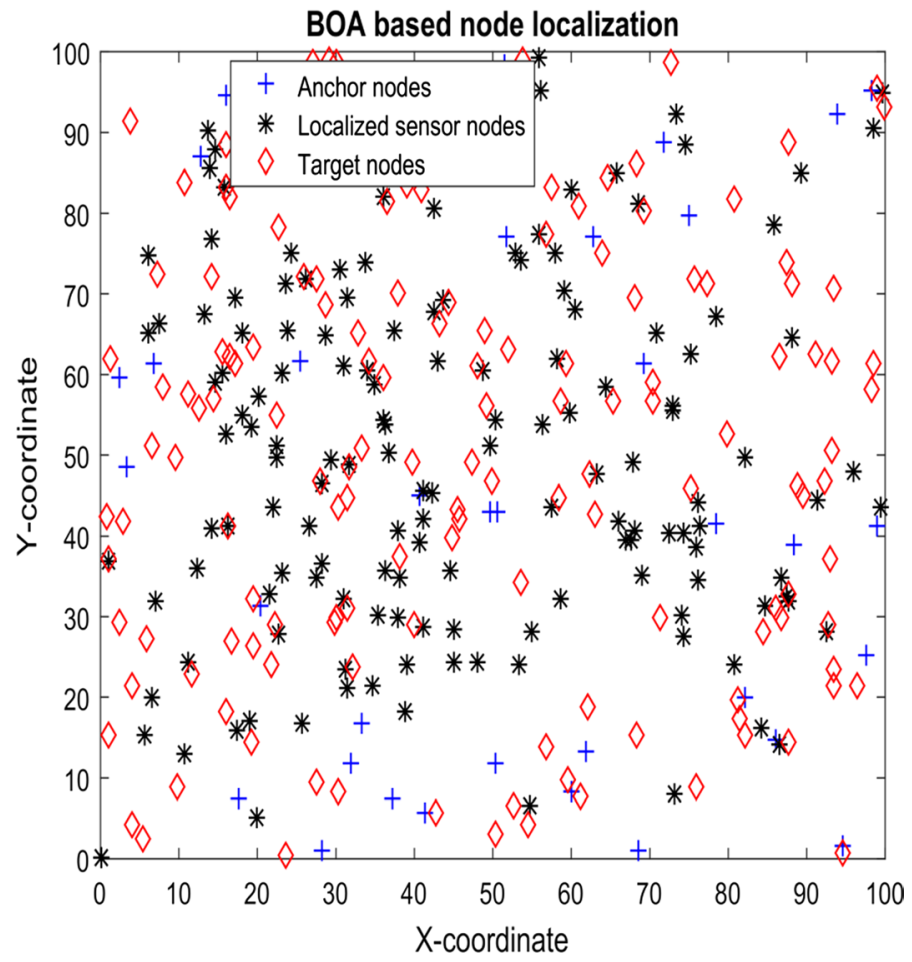

Fig. 2 Node localization based on BOA in WSNs

In addition to this, proposed BOA variants 1 and 2 are also compared with other optimization algorithms which are already applied to localization problem in WSNs such as particle swarm optimization (PSO) algorithm [49], firefly algorithm (FA) [49], butterfly optimization algorithm (BTOA) [49], salp swarm optimization (SSO) algorithm [49] and grey wolf optimization (GWO) algorithm [49]. The performance of the proposed BOA variants 1 and 2, BOA, FA, BTOA, PSO, GWO and SSA is estimated for various scenarios of target nodes and anchor nodes in terms of number of localized nodes $\left(N_{L}\right)$, computation time $(T(s))$ and mean localization error $(M L E)$. The results achieved from the various optimization algorithms for node localization in terms of $N_{L}$ and $T(s)$ are shown in Table 5, and $M L E$ of numerous optimization algorithms is revealed in Table 6 , respectively.

From Tables 5 and 6 , it is clear that with an increase in the number of iterations all optimization algorithms such as PSO, GWO, BTOA, FA, SSA and original BOA localized more number of sensor nodes, which in turn decreases the mean localization error, and computation time is increased for different numbers of target nodes and anchor nodes. With an increase in iterations, each of these algorithms has to perform more calculations which in turn increase the computing time of algorithms. When the iterations are increased, each of these algorithms has more chances to discover the best optimum solution, and therefore these algorithms localized more nodes and mean localization error decreased. 


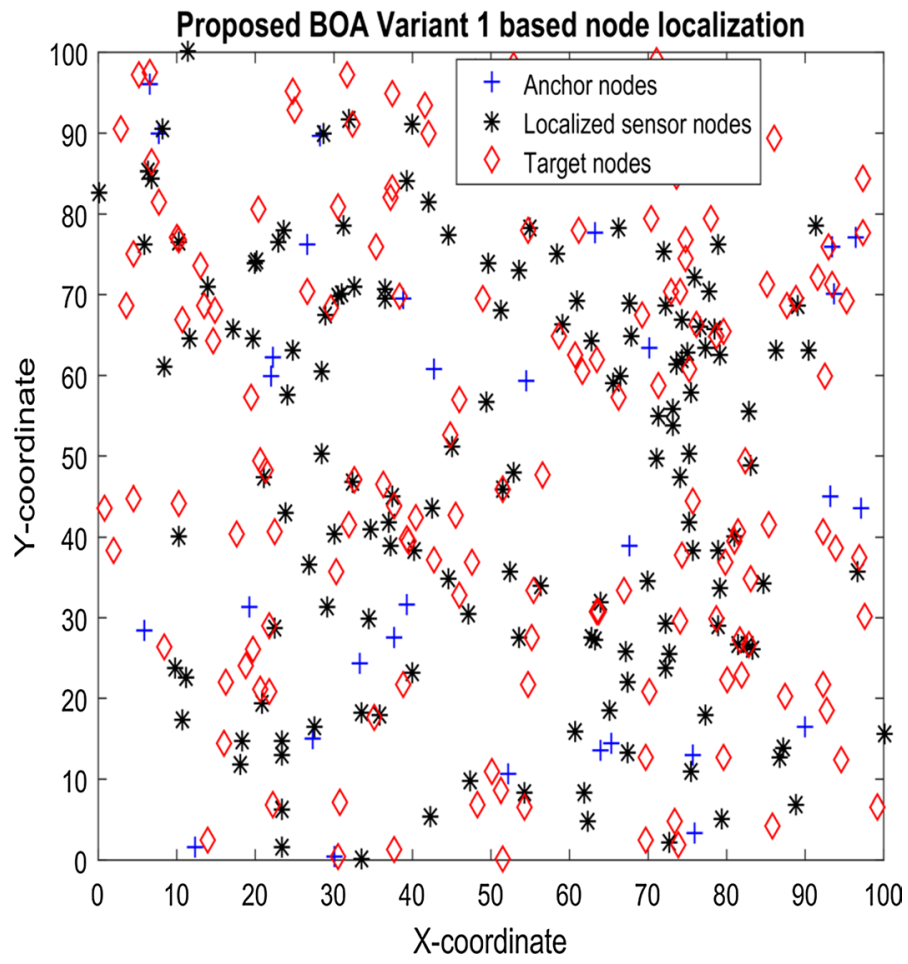

Fig. 3 Node localization based on proposed BOA variant 1 in WSNs

But in the proposed BOA variants 1 and 2, the search space is large compared to these algorithms. The proposed BOA variants 1 and 2 localized all the target nodes at 25 iterations for various scenarios of target nodes and anchor nodes. The value of mean localization error is less for the proposed BOA variants 1 and 2 at 25 iterations for various target nodes and anchor nodes. With an increase in iterations, both mean localization error and computing time increase for the proposed BOA variants 1 and 2 as shown in Tables 5 and 6 . The proposed BOA variants 1 and 2 have improved exploration and exploitation abilities and converged at earlier stage. By increasing the iterations, the proposed BOA variants 1 and 2 move away from the current best solution in order to find better best solution which increases the distance between estimated location and actual position of nodes; as a result, mean localization error increases. The mean localization error increases because the search area of both the proposed BOA variants 1 and 2 is already enhanced by introducing improved local and global search strategies and BOA variants 1 and 2 have discovered optimum location of nodes at earlier stages.

The summary of best results of these optimization algorithms and the proposed BOA variants 1 and 2 in terms of $N_{L}$ and $T(s)$ is represented in Table 7 and in terms of $M L E$ is shown in Table 8, respectively. The mean localization error and number of localized nodes vs various number of target nodes and anchor nodes for several optimization algorithms SSA, FA, BTOA, PSO, GWO, BOA and proposed BOA 


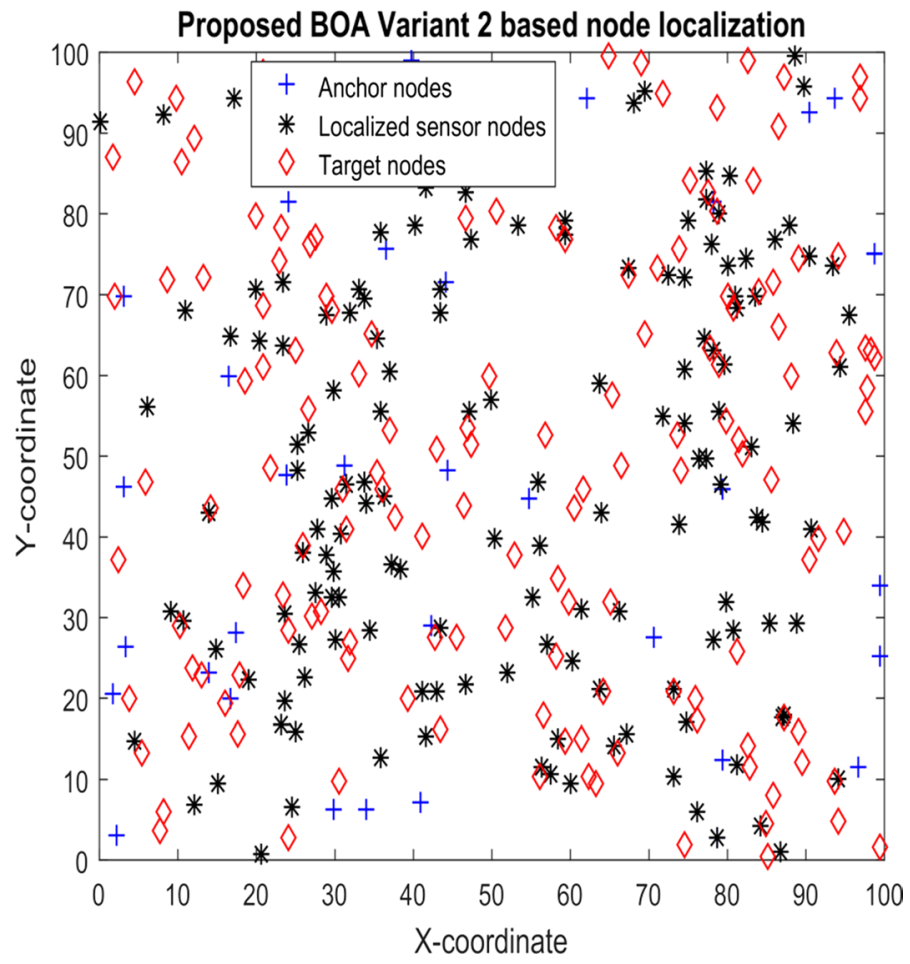

Fig. 4 Node localization based on proposed BOA variant 2 in WSNs

variants 1 and 2 are also shown in Figs. 5 and 6, respectively. The algorithms such as PSO, FA, BTOA, SSA, GWO and original BOA provide the minimum value of mean localization error and localized more sensor nodes at 100 iterations. The proposed BOA variants 1 and 2 localized all target nodes and provide less mean localization error at 25 iterations. From Tables 7 and 8, it is obvious that original BOA performs superior to PSO, FA, GWO and SSA in terms of computation time and mean localization error, but it does not localize all target nodes in the network due to random deployment of sensor nodes.

It is clear from Fig. 5 and Table 8 that the mean localization error of BTOA is less compared to original BOA until target nodes and anchor nodes are equal to 100 and 25. When the value of target nodes and anchor nodes increases beyond these values, the mean localization error of BOA is less than that of BTOA. The BTOA algorithm takes more computation time than the existing BOA as shown in Table 7.

To overcome the drawback of the existing bat algorithm, BOA variant 1 is proposed. From Table 7, it is obvious that the proposed BOA variant 1 has smaller computation time than other algorithms PSO, SSA, BTOA, FA, GWO and existing BOA for various values of target nodes and anchor nodes. The value of mean localization error of the proposed BOA variant 1 is lowest compared to the above-stated existing optimization algorithms and BOA for several set-ups of target and anchor nodes as shown in Fig. 5 and Table 8. The proposed BOA variant 1 has localized all the target 


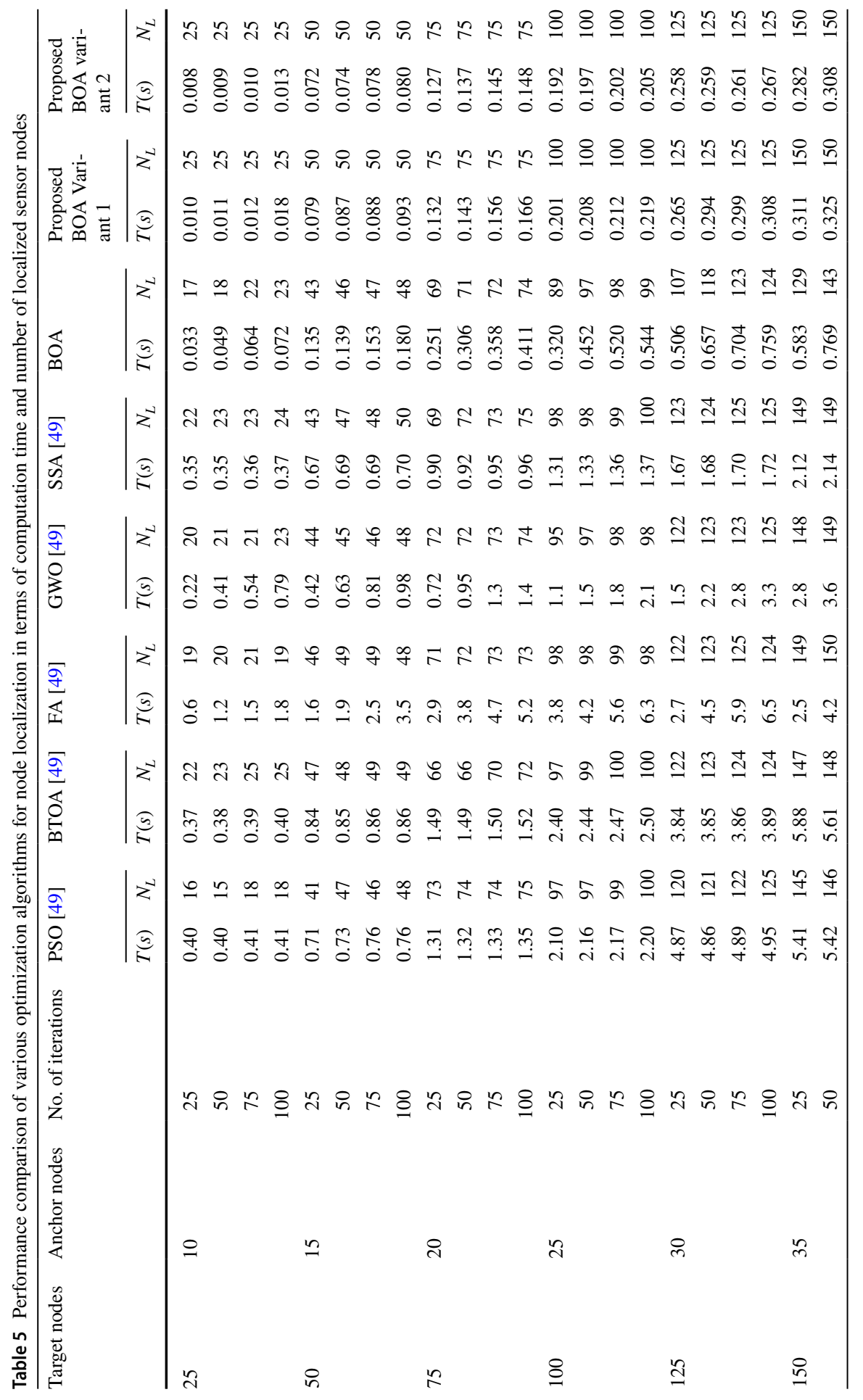




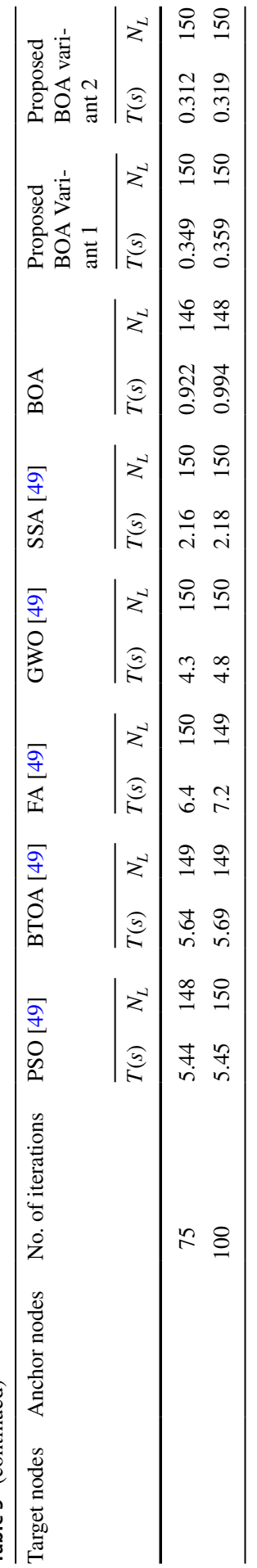




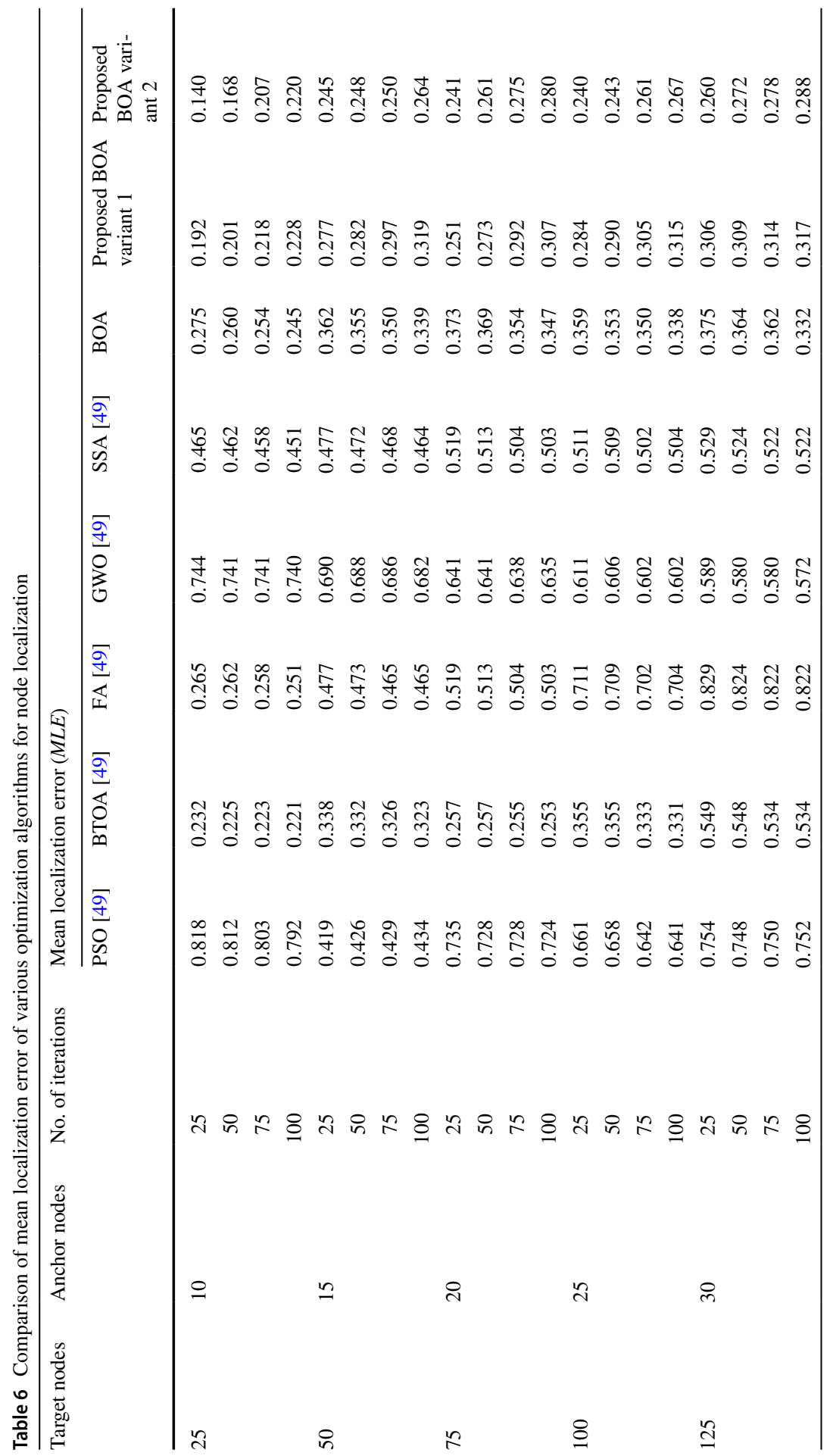




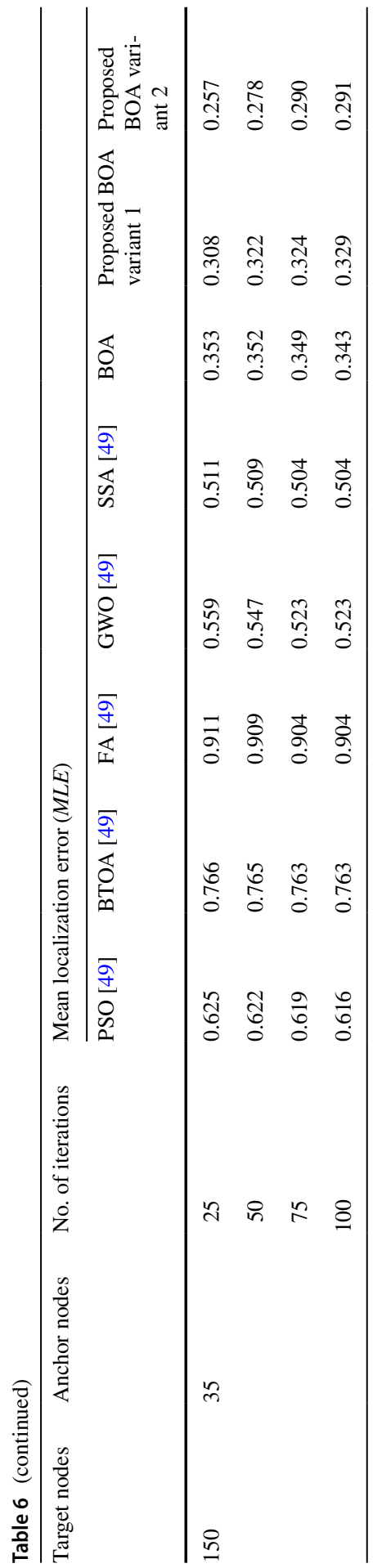




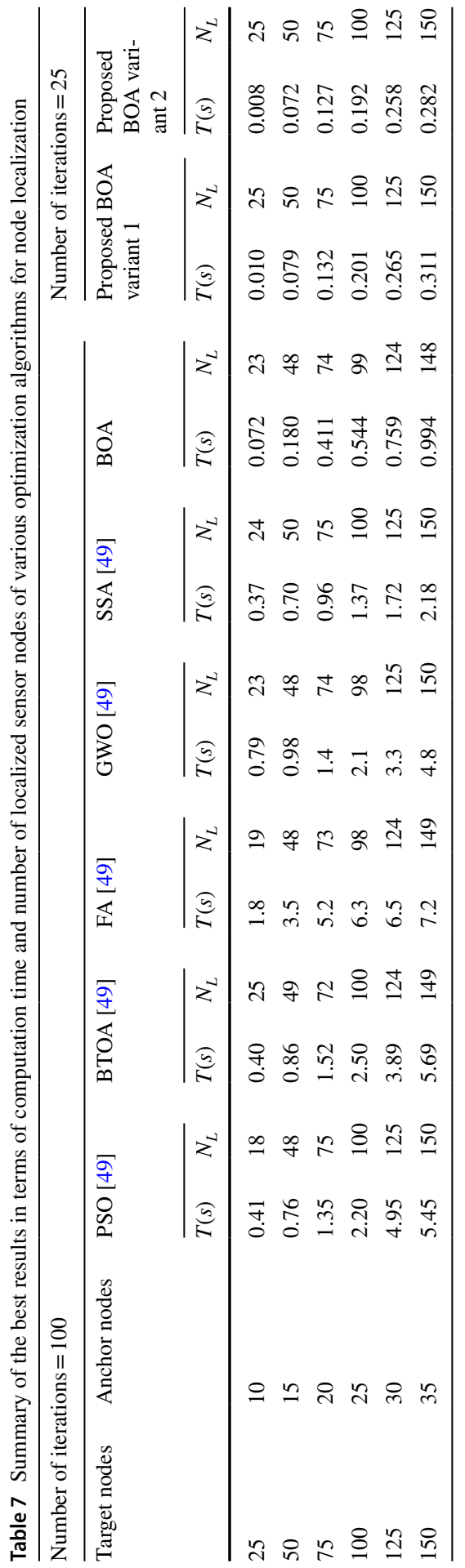




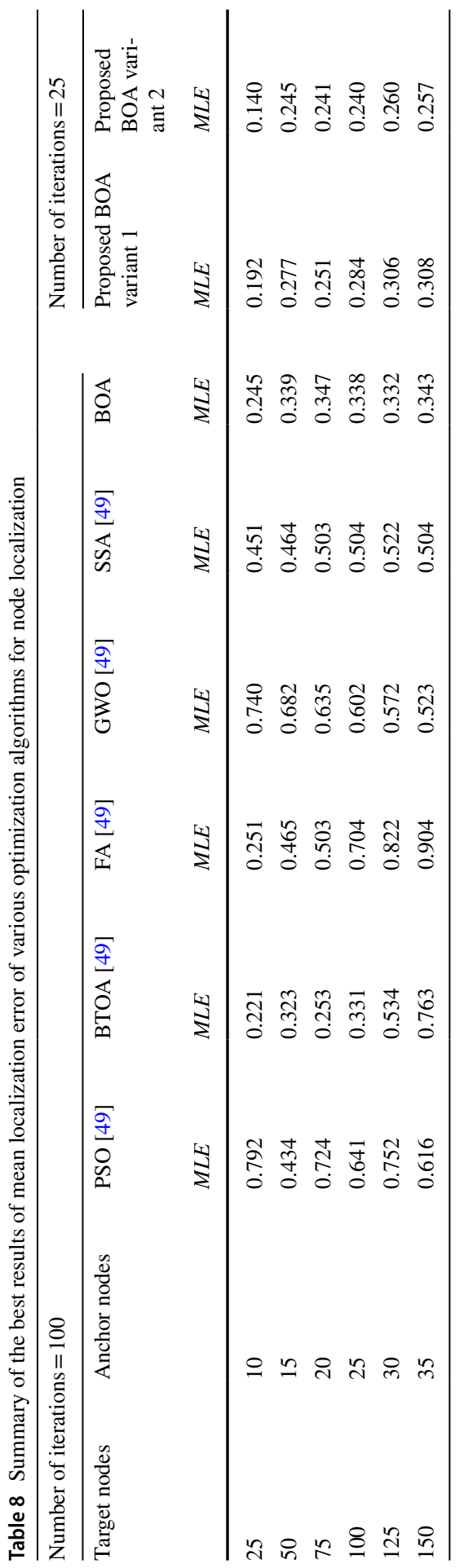




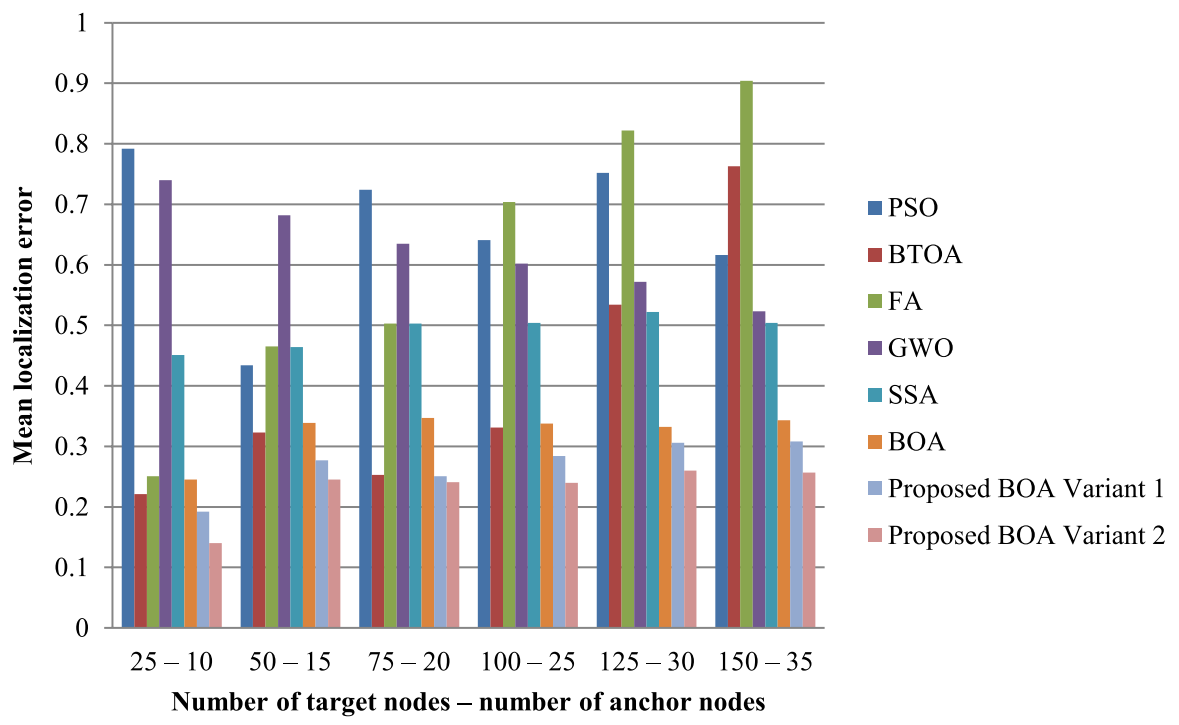

Fig. 5 Mean localization error of various optimization algorithms used for node localization for different scenarios

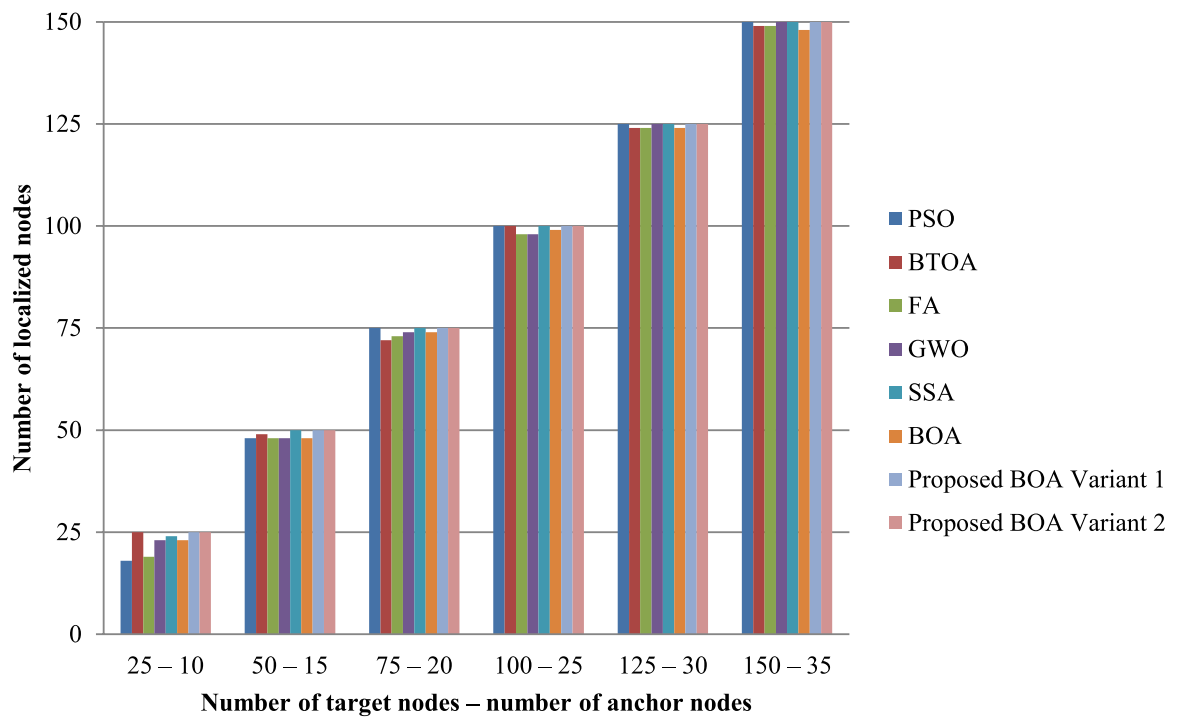

Fig. 6 Number of localized nodes of various optimization algorithms used for node localization for different scenarios 
nodes in WSNs at earlier stage, i.e. at 25 iterations for each scenario of target nodes and anchor nodes as revealed in Fig. 6 and Table 7.

The computation time of the proposed BOA variant 1 is decreased by $68.7 \%$, $85.7 \%, 93.5 \%, 94.3 \%, 94.5 \%$ and $95.6 \%$ than that of original BOA, SSA, GWO, PSO, BTOA and FA, respectively, for 150 target nodes and 25 anchor nodes scenario as shown in Table 7. It can be observed from Table 8 that for 150 target nodes and 25 anchor nodes scenario the mean localization error of the proposed BOA variant 1 is reduced by $10.2 \%, 38.8 \%, 41.1 \%, 50 \%, 59.6 \%$ and $65.9 \%$ than original BOA, SSA, GWO, PSO, BTOA and FA, respectively.

In order to further reduce the localization error and computation time, BOA variant 2 is introduced. The proposed BOA variant 2 outperforms other algorithms FA, PSO, GWO, BTOA, SSA, original BOA and proposed BOA variant 1 in terms of computation time, localized nodes and mean localization error for various cases of anchor nodes and target nodes as revealed in Tables 7 and 8. The mean localization error of the proposed BOA variant 2 is less than other optimization algorithms such as BTOA, PSO, FA, SSA, GWO, original BOA and proposed BOA variant 1 for each scenario of target nodes and anchor nodes as shown in Fig. 5 and Table 8. The proposed BOA 2 also converged at 25 iterations, localized all target nodes at initial stage, i.e. 25 iterations, and has smallest computation time than these existing optimization algorithms, BOA and proposed BOA variant 1 for various scenarios of anchor nodes and target nodes as shown in Fig. 6 and Table 7.

It can be seen from Table 7 that the computation time of the proposed BOA variant 2 is decreased by $9.3 \%, 71.6 \%, 87 \%, 94.1 \%, 94.8 \%, 95 \%$ and $96 \%$ than that of the proposed BOA variant 1, original BOA, SSA, GWO, PSO, BTOA and FA, respectively, for 25 anchor nodes and 150 target nodes scenario. The mean localization error of the proposed BOA variant 2 is reduced by $16.6 \%, 25 \%, 49 \%, 50.8 \%, 58.2 \%$, $66.3 \%$ and $71.5 \%$ than the proposed BOA variant 1, original BOA, SSA, GWO, PSO, BTOA and FA, respectively, for 25 anchor nodes and 150 target nodes scenario as revealed in Table 8.

\subsection{Computational analysis of the proposed BOA variants 1 and 2 in terms of different types of errors and localization efficiency}

Moreover, the existing BOA and proposed BOA variants 1 and 2 are also compared in terms of localization efficiency $(L E(\%))$, average localization error $(A L E)$, normalized localization error $(N L E(\%))$ and root-mean-square error (RMSE) as shown in Table 9. To reduce the influence of randomly deployed sensor nodes, BOA and the proposed BOA variants 1 and 2 are executed 50 times, and the best results are selected. The localization efficiency of the proposed BOA variants 1 and 2 is $100 \%$ for different values of target nodes, anchor nodes and iterations as shown in Table 9. From Table 9, it is observable that the average localization error, RMSE and normalized localization error for existing BOA decrease with an increase in the number of iterations. When iterations are increased, the number of localized nodes increases, which in turn increases the localization efficiency and decreases the ALE, RMSE and $\operatorname{NLE}(\%)$. 


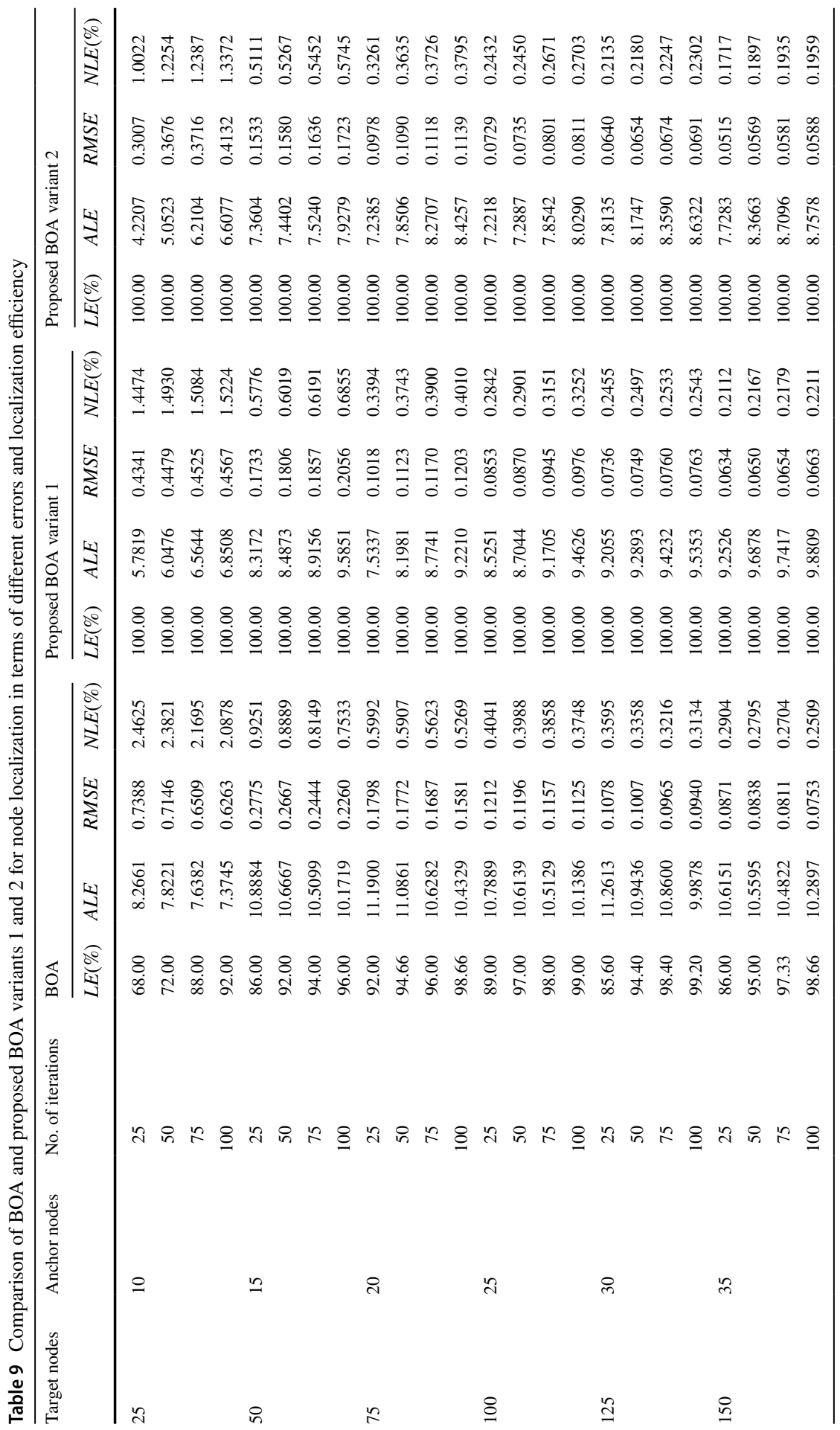


From Table 9, it is evident that the proposed BOA variants 1 and 2 converge at 25 iterations, while original BOA converges at 100 iterations. The average localization error, normalized localization error and root-mean-square error of the proposed BOA variant 1 and variant 2 are increased with an increase in number of iterations as shown in Table 9. By increasing the iterations, the proposed BOA variants 1 and 2 deviate from the best optimum value to search more optimum value which in turn increases the $A L E, N L E(\%)$ and $R M S E$ for the proposed BOA variants 1 and 2.

The summary of optimum values of average localization error, localization efficiency, root-mean-square error and normalized localization error is presented in Table 10. The localization efficiency, average localization error, root-mean-square error and normalized localization error vs number of target nodes and anchor nodes for BOA and proposed BOA variants 1 and 2 are shown in Figs. 7, 8, 9 and 10, respectively.

From Table 10 and Fig. 7, it is easily seen that localization efficiency of existing BOA algorithm is less compared to proposed BOA variants 1 and 2 for different scenarios of target nodes and anchor nodes. The average localization error, root-meansquare error and normalized localization error of the proposed BOA variant 1 are less than original BOA, but they are higher than the proposed BOA variant 2 for various cases of target and anchor nodes as represented in Table 10 and Figs. 8, 9 and 10, respectively.

For 150 target nodes and 25 anchor nodes scenario, the accuracy of the proposed BOA variants 1 and 2 is improved by $10 \%$ and $24.8 \%$, respectively, than original BOA. In other words, the average localization error of the proposed BOA variants 1 and 2 for 150 target nodes and 25 anchor nodes is decreased by $10 \%$ and $24.8 \%$, respectively, compared to BOA as shown in Table 10. The root-mean-square error and normalized localization error of the proposed BOA variants 1 and 2 are reduced by $15.8 \%$ and $31.6 \%$, respectively, than those of BOA for 150 target nodes and 25 anchor nodes scenario as revealed in Table 10. The proposed BOA variant 2 has less root-mean-square error, average localization error and normalized localization error than that of the proposed BOA variant 1 and existing BOA for different scenarios as exposed in Table 10 and Figs. 7, 8, 9 and 10, respectively. Thus, the proposed BOA variant 2 is superior to the proposed BOA variant 1 and original BOA in terms of root-mean-square error, average localization error and normalized localization error for various values of target and anchor nodes.

\subsection{Effect of anchor nodes on performance of BOA and proposed BOA variants 1 and 2}

The number of localizable nodes and localization accuracy depend on the number of anchor nodes deployed in the wireless sensor network. It is a very challenging task to localize the nodes in the network if required number of nodes, i.e. at least three anchor nodes, does not lie in the range of target node. The performance of optimization algorithm used for node localization depends on the number of anchor nodes. The effect of anchor nodes on $N_{L}, T(s)$ and $M L E$ of BOA and proposed BOA variants 1 and 2 is shown in Table 11, and Table 12 signifies the influence of anchor 
node on $L E(\%), A L E, R M S E, N L E(\%)$ of BOA and proposed BOA variants 1 and 2, respectively. In Tables 11 and 12, the anchor nodes are varied from 10 to 50, while other parameters such as range of sensor nodes, monitoring area, target nodes, ranging error and maximum iterations are set to $30 \mathrm{~m}, 100 \mathrm{~m} \times 100 \mathrm{~m}, 150,2 \%$ and 100 , respectively, for BOA and the proposed BOA variants 1 and 2. The BOA and the proposed BOA variants 1 and 2 are simulated 50 times to reduce the effect of randomness, and the best values of results are chosen. The effect of the number of anchor nodes on various parameters such as computing time, localization efficiency, average localization error, mean localization error, root-mean-square error and normalized localization error of BOA and the proposed BOA variants 1 and 2 is shown in Figs. 11, 12, 13, 14, 15 and 16, respectively.

There is a contradiction between different types of localization error and cost of the network. If more number of anchor nodes are used in the network, the cost of the whole network will be high because the location of anchor nodes is determined with the help of GPS. By using 20 anchor nodes for both proposed BOA variants 1 and 2, total target nodes, i.e. 150 nodes, are localized, but the value of $A L E, M L E$, $N L E(\%)$ and RMSE is high compared to the values of these errors at 35 anchor nodes as shown in Tables 11 and 12. The original BOA localized 143 target nodes for 20 anchor nodes as revealed in Table 11, and for each value of anchor nodes, the values of various errors such as MLE,ALE, RMSE and $N L E(\%)$ of BOA are higher than that of the proposed BOA variants 1 and 2 as shown in Tables 11 and 12, respectively. The performance of the proposed BOA variants 1 and 2 is better at 35 anchor nodes compared to 20 anchor nodes because values of various errors are less, but increase the cost of the whole network. The proposed BOA variants 1 and 2 are cost-effective for 20 anchor nodes, and they localized all target nodes.

From Table 11, it is clear that for 20 anchor nodes the $M L E$ of the proposed BOA variant 1 is 0.3756 which is less than all existing algorithms such as FA, PSO, BTOA, SSA and GWO which are using 35 anchor nodes as revealed in Table 5 and it is also less than MLE of BOA for 20 anchor nodes as shown in Table 11. The MLE of existing algorithms such as FA, GWO, PSO, SSA and BTOA will be higher than that of the proposed BOA variants 1 and 2 for 20 anchor nodes because small anchor nodes will localize less target nodes in WSN which results in high localization error. As shown in Table 11, for 20 anchor nodes the $M L E$ of the proposed BOA variant 2 is 0.3182 which is less than all existing algorithms such as FA, PSO, BTOA, SSA, GWO which are using 35 anchor nodes as shown in Table 5. Thus, for 20 anchor nodes the proposed BOA variant 2 is more effective than the proposed BOA variant 1 and BOA because the value of $M L E$ of the proposed BOA variant 2 is less than that of the proposed BOA variant 1 and BOA. Therefore, in order to make cost-effective WSN and to localize all unknown nodes, the optimized value of anchor nodes is fixed to 20 for both proposed BOA variants 1 and 2 .

The computation time, $L E(\%), A L E, M L E, R M S E$ and $N L E(\%)$ of the proposed BOA variant 2 are less than those of the proposed BOA variant 1 and BOA for various numbers of anchor nodes as shown in Figs. 11, 12, 13, 14, 15 and 16, respectively. So the proposed BOA variant 2 is more efficient than the proposed BOA variant 1 and $\mathrm{BOA}$ in terms of computing time, various errors stated above and 


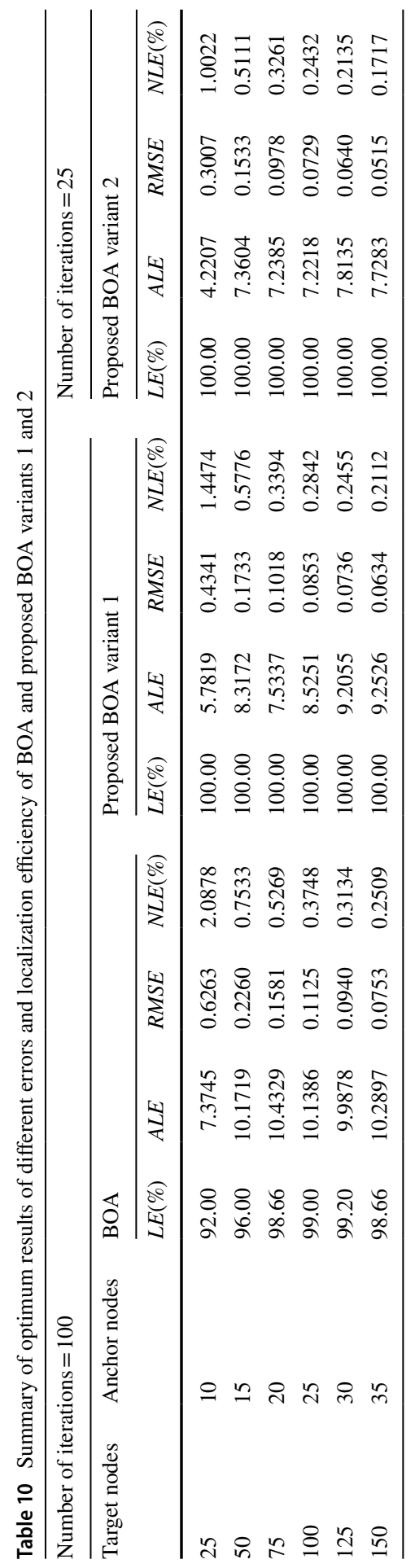




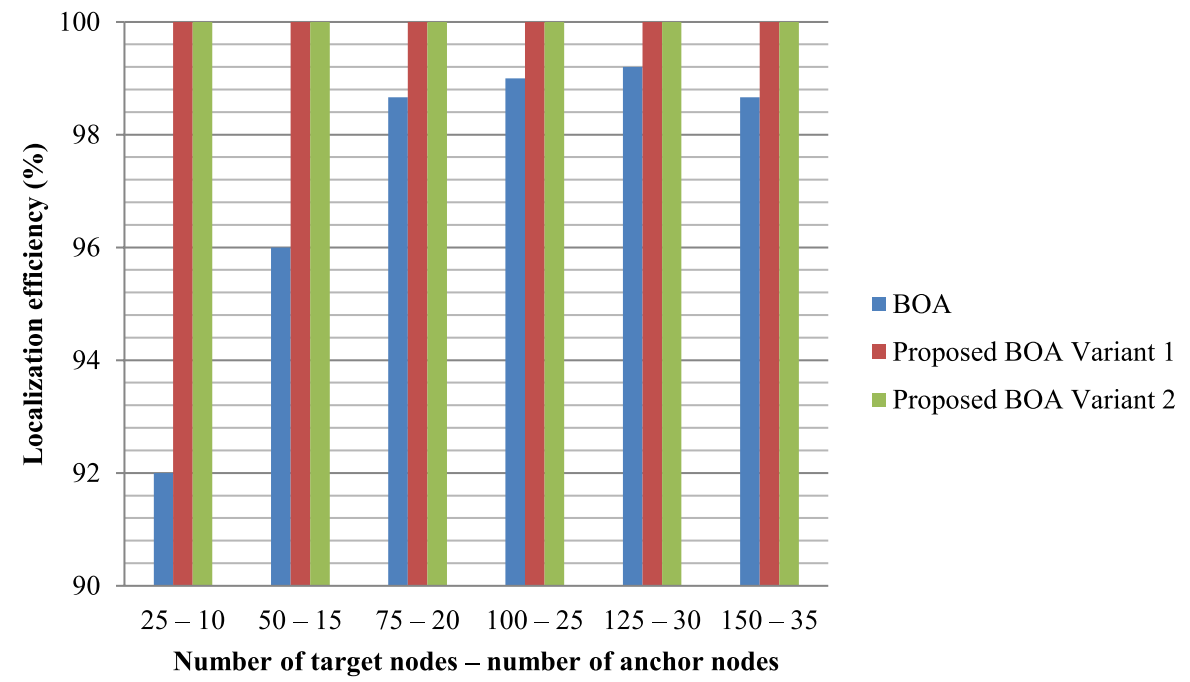

Fig. 7 Localization efficiency of BOA, proposed BOA variants 1 and 2 for different scenarios

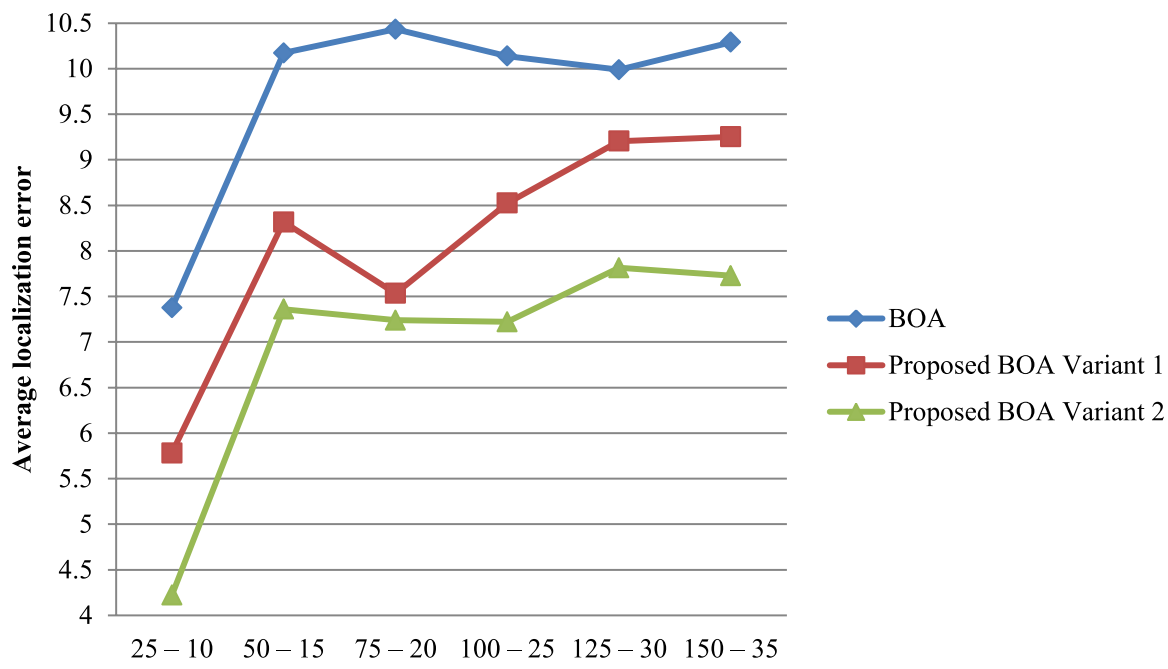

Number of target nodes - number of anchor nodes

Fig. 8 Average localization error of BOA, proposed BOA variants 1 and 2 for different scenarios

localization efficiency for various numbers of anchor nodes as revealed in Tables 11 and 12 respectively. 


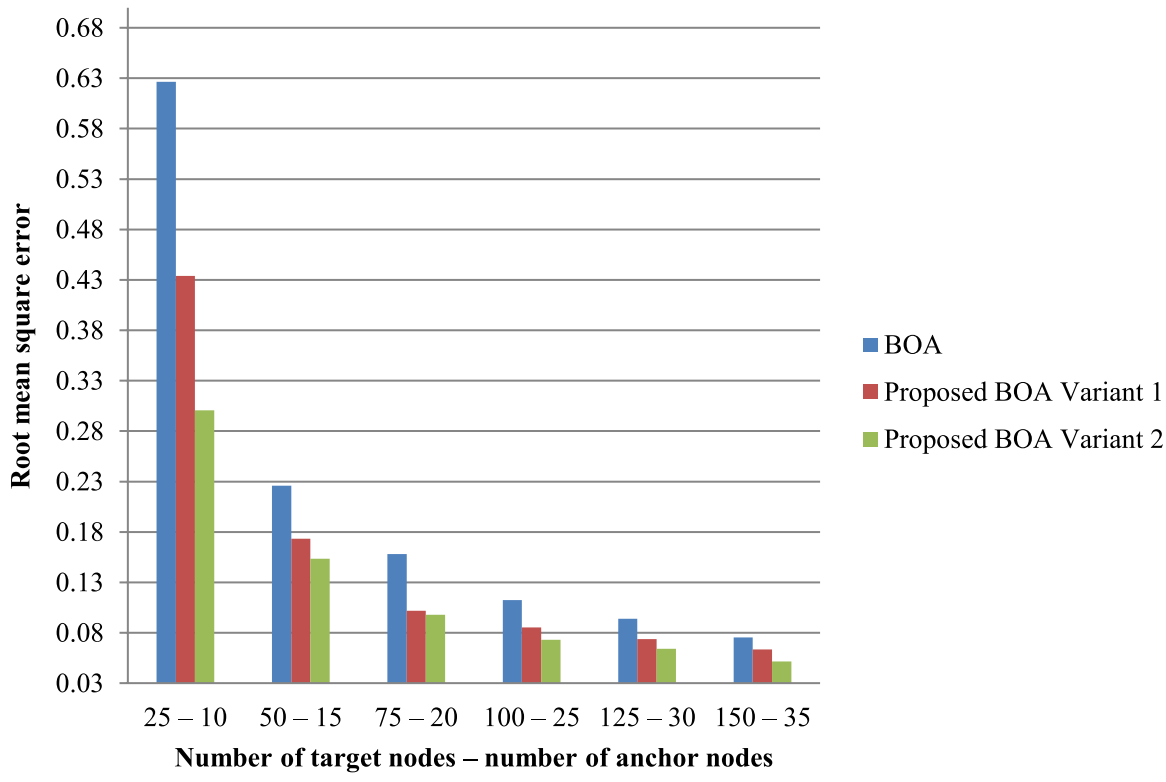

Fig. 9 Root-mean-square error of BOA, proposed BOA variants 1 and 2 for different scenarios

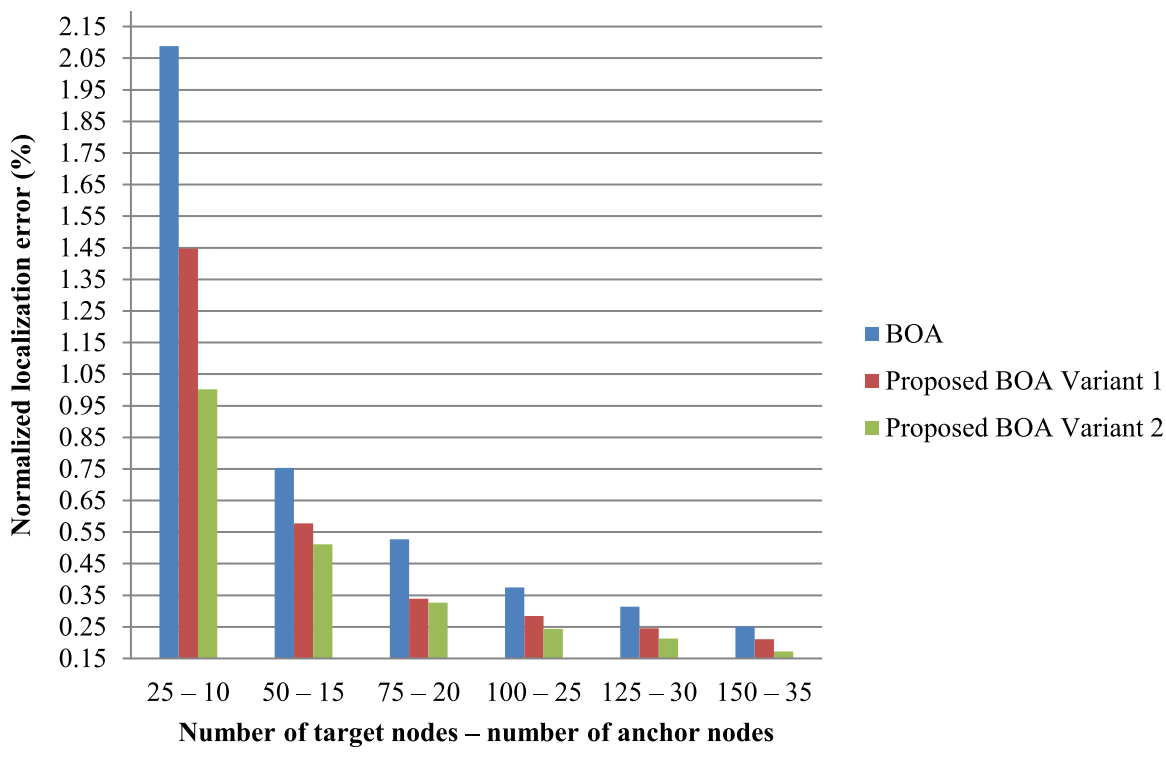

Fig. 10 Normalized localization error (\%) of BOA, proposed BOA variants 1 and 2 for different scenarios 
Table 11 Influence of anchor nodes on computation time, mean localization error and number of localized nodes of BOA and proposed BOA variants 1 and 2

Transmission range $R=30 \mathrm{~m}$, monitoring area $=100 \mathrm{~m} \times 100 \mathrm{~m}$, target nodes $N=150$, maximum iterations $M I=100$, ranging error $=2 \%$

\begin{tabular}{|c|c|c|c|c|c|c|c|c|c|}
\hline \multirow[t]{2}{*}{ Anchor nodes } & \multicolumn{3}{|l|}{$\mathrm{BOA}$} & \multicolumn{3}{|c|}{ Proposed BOA variant 1} & \multicolumn{3}{|c|}{ Proposed BOA variant 2} \\
\hline & $T(s)$ & $N_{L}$ & $M L E$ & $T(s)$ & $N_{L}$ & $M L E$ & $T(s)$ & $N_{L}$ & $M L E$ \\
\hline 10 & 0.9574 & 131 & 0.4768 & 0.3475 & 144 & 0.4479 & 0.2979 & 146 & 0.3278 \\
\hline 15 & 0.9652 & 134 & 0.4209 & 0.3564 & 148 & 0.4044 & 0.3134 & 149 & 0.3252 \\
\hline 20 & 0.9730 & 143 & 0.3955 & 0.3615 & 150 & 0.3756 & 0.3149 & 150 & 0.3182 \\
\hline 25 & 0.9808 & 145 & 0.3695 & 0.3662 & 150 & 0.3409 & 0.3157 & 150 & 0.3108 \\
\hline 30 & 0.9950 & 146 & 0.3585 & 0.3713 & 150 & 0.3334 & 0.3164 & 150 & 0.3081 \\
\hline 35 & 1.0099 & 148 & 0.3454 & 0.3726 & 150 & 0.3255 & 0.3182 & 150 & 0.2922 \\
\hline 40 & 1.0175 & 149 & 0.3379 & 0.3746 & 150 & 0.3245 & 0.3221 & 150 & 0.2872 \\
\hline 45 & 1.0248 & 150 & 0.3285 & 0.3757 & 150 & 0.3210 & 0.3324 & 150 & 0.2836 \\
\hline 50 & 1.0352 & 150 & 0.3177 & 0.3786 & 150 & 0.3030 & 0.3329 & 150 & 0.2794 \\
\hline
\end{tabular}

\subsection{Effect of monitoring area on performance of BOA, proposed BOA variants 1 and 2}

In order to observe the effect of monitoring area on the performance of BOA and the proposed BOA variants 1 and 2 , the monitoring area is varied from $100 \mathrm{~m} \times 100 \mathrm{~m}$ to $400 \mathrm{~m} \times 400 \mathrm{~m}$, respectively. The values of other parameters such as anchor nodes, target nodes, transmission range and ranging error are selected as shown in Table 13 for various values of monitoring area. When the size of monitoring area is increased, the effect of noise is also increased; therefore, the value of noise (ranging error) is also increased as shown in Table 13.

Table 14 signifies the effect of monitoring area on the performance of BOA and proposed BOA variants 1 and 2. For BOA and the proposed BOA variants 1 and 2, the number of localized nodes $\left(N_{L}\right)$ and localization efficiency $(L E(\%))$ are computed for each value of monitoring area as shown in Table 14. To analyse the performance of $\mathrm{BOA}$ and proposed BOA variants 1 and 2, the maximum iterations are varied from 25 to 100 as shown in Table 14. The target nodes and anchor nodes are deployed arbitrarily in the monitoring area. To reduce the influence of arbitrariness, each algorithm of BOA and the proposed BOA variants 1 and 2 is run 50 times and the best values of $N_{L}$ and $L E(\%)$ are selected.

It can be seen from Table 14 for $100 \mathrm{~m} \times 100 \mathrm{~m}$ monitoring area the proposed BOA variants 1 and 2 localized all target nodes at 25 iterations, but BOA localized 148 target nodes at 100 iterations. Therefore, the proposed BOA variants 1 and 2 converge at 25 iterations and BOA converges at 100 iterations for $100 \mathrm{~m} \times 100 \mathrm{~m}$ monitoring area. When the size of monitoring area is increased to $200 \mathrm{~m} \times 200 \mathrm{~m}$, the BOA localized only 231 nodes at 100 iterations and the proposed BOA variant 1 localized 297 target nodes at 25 iterations. For $200 \mathrm{~m} \times 200 \mathrm{~m}$ monitoring area, the proposed BOA variant 1 converges at 50 iterations and localized all 


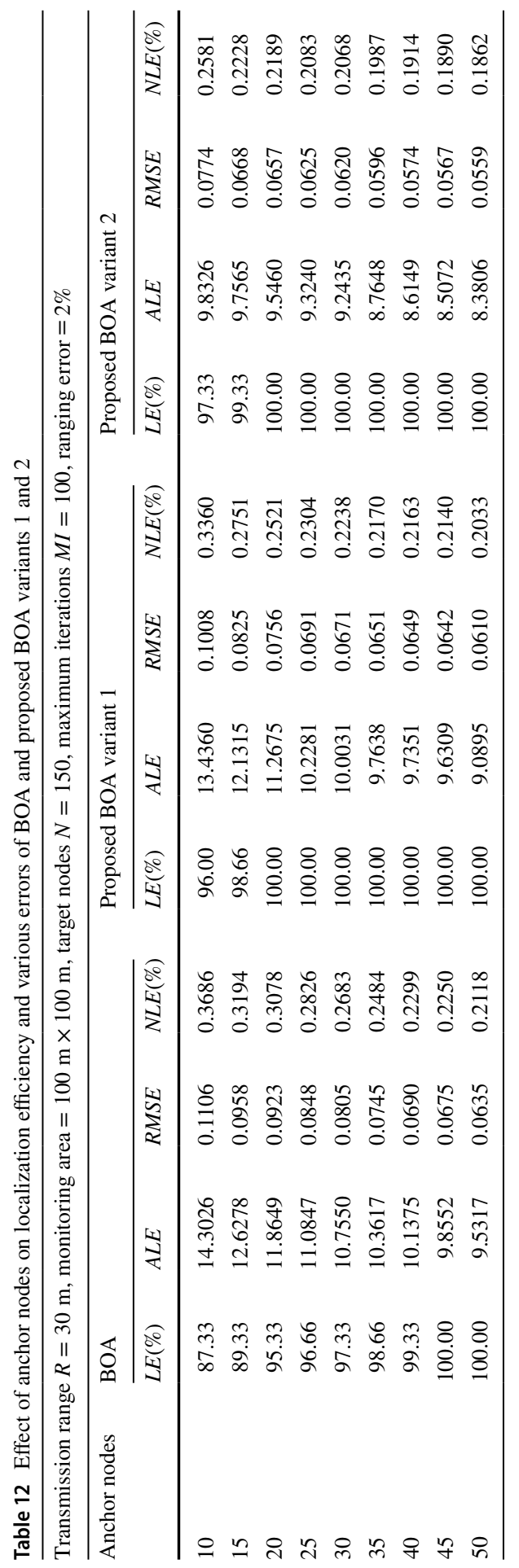




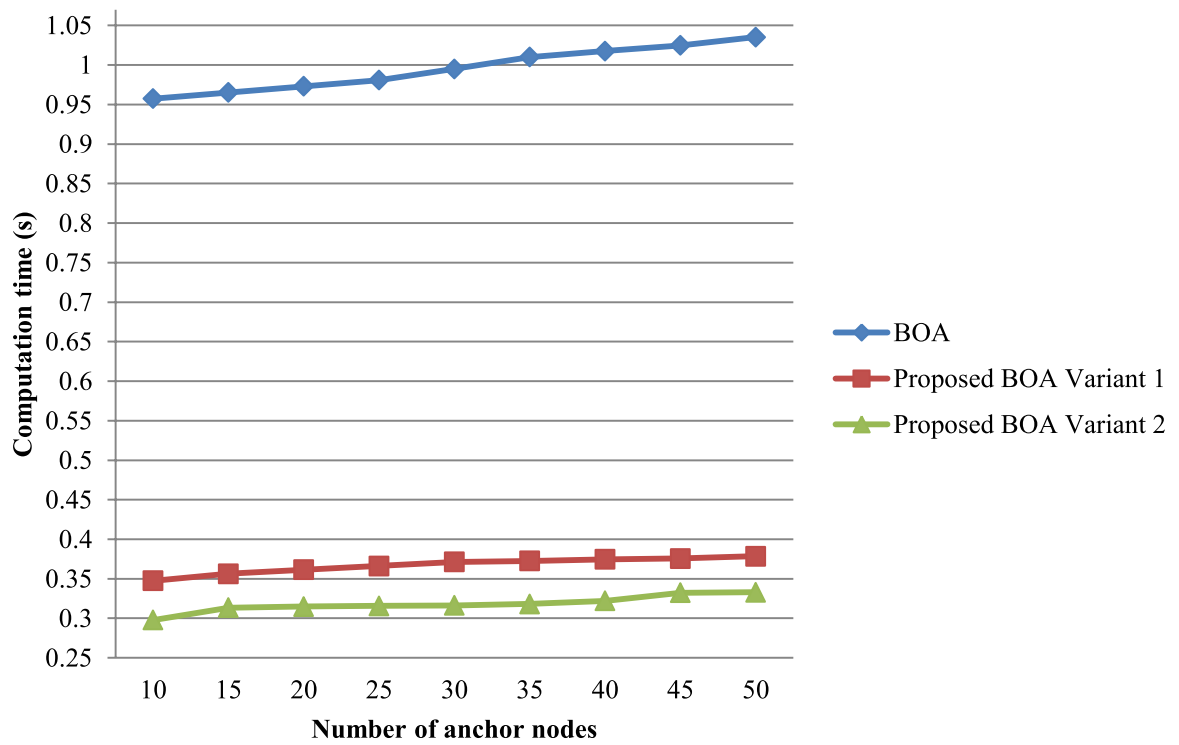

Fig. 11 Computation time of BOA, proposed BOA variants 1 and 2 for various numbers of anchor nodes

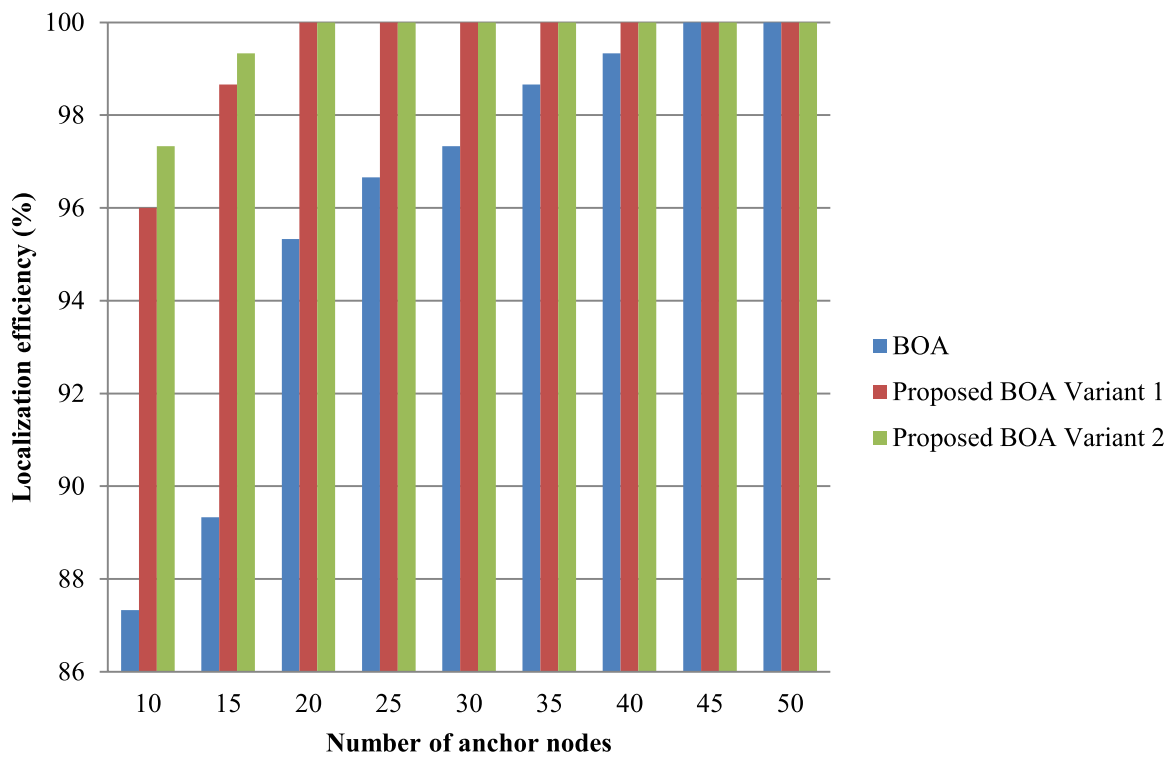

Fig. 12 Localization efficiency (\%) of BOA, proposed BOA variants 1 and 2 for various numbers of anchor nodes

target nodes in the network as shown in Table 14. It can be observed that the proposed BOA variant 2 localized all target nodes and converges at 25 iterations for $200 \mathrm{~m} \times 200 \mathrm{~m}$ monitoring area. 


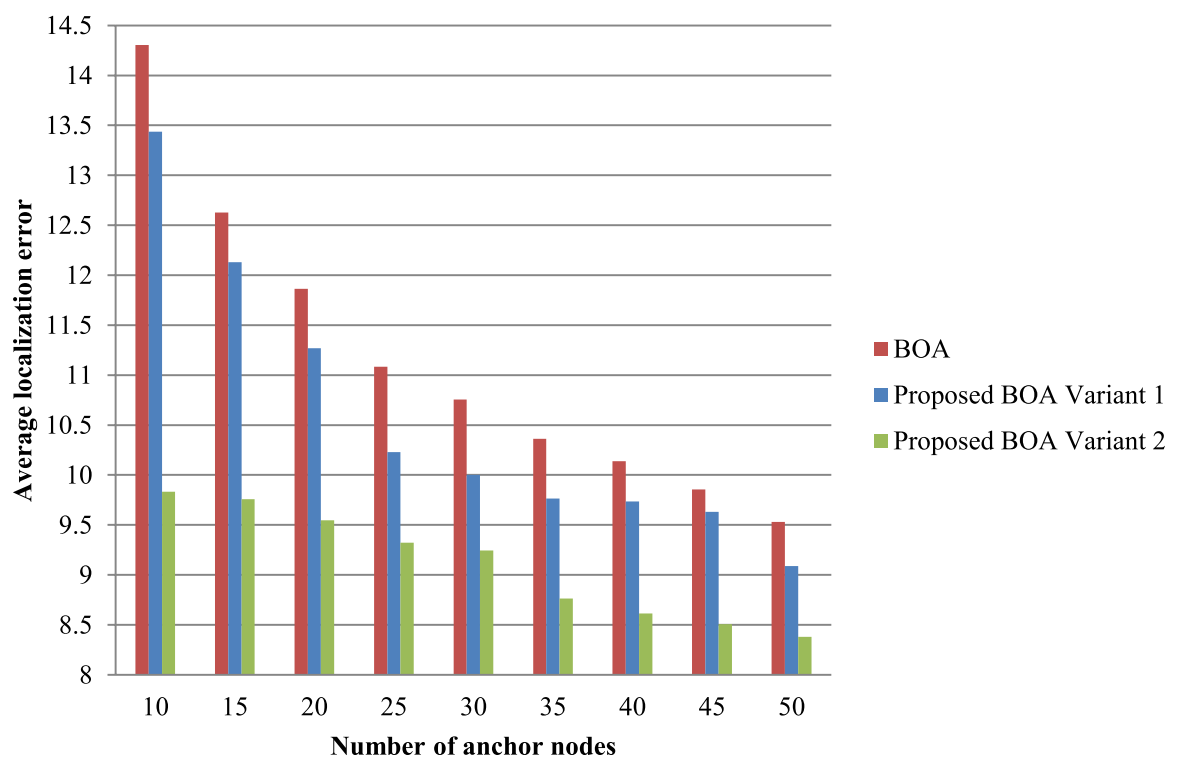

Fig. 13 Average localization error of BOA, proposed BOA variants 1 and 2 for various numbers of anchor nodes

Similarly, when the size of monitoring area is further increased to $300 \mathrm{~m} \times 300 \mathrm{~m}$ and $400 \mathrm{~m} \times 400 \mathrm{~m}$ BOA does not localize all target nodes at 100 iterations, the proposed BOA variant 1 localized all target nodes at 50 iterations and the proposed BOA variant 2 localized all target nodes at 25 iterations as revealed in Table 14. Therefore, when the size of monitoring area is increased the proposed BOA variant 2 converges at 25 iterations and the proposed BOA variant 1 converges at 50 iterations. Thus, the proposed BOA variant 2 converges faster than the proposed BOA variant 1 and BOA when the size of monitoring area is increased. But the proposed BOA variant 1 does not localize all target nodes at 25 iterations when the size of monitoring area is increased and localization efficiency of the proposed BOA variant 1 is not $100 \%$ at 25 iterations. The proposed BOA variant 1 localized all target nodes at 50 iterations that slows the convergence rate of the proposed BOA variant 1 when the size of monitoring area is increased beyond $100 \mathrm{~m} \times 100 \mathrm{~m}$. The proposed BOA variant 2 is scalable, meaning that it localized all target nodes in the WSN at 25 iterations when monitoring area is increased. The performance of the proposed BOA variant 2 is not affected by the size of monitoring area.

To compare the localization efficiency of BOA and the proposed BOA variants 1 and 2 , the number of iterations is fixed to 25 and localization efficiency vs monitoring area is plotted in Fig. 17. The localization efficiency of the proposed BOA variants 1 and 2 is $100 \%$ for $100 \mathrm{~m} \times 100 \mathrm{~m}$. It can be seen from Fig. 17 that the localization efficiency of BOA decreases with an increase in the size of monitoring area at 25 iterations. The localization efficiency of the proposed BOA variant 2 is higher than that of the proposed BOA variant 1 and BOA when the size of monitoring area is 


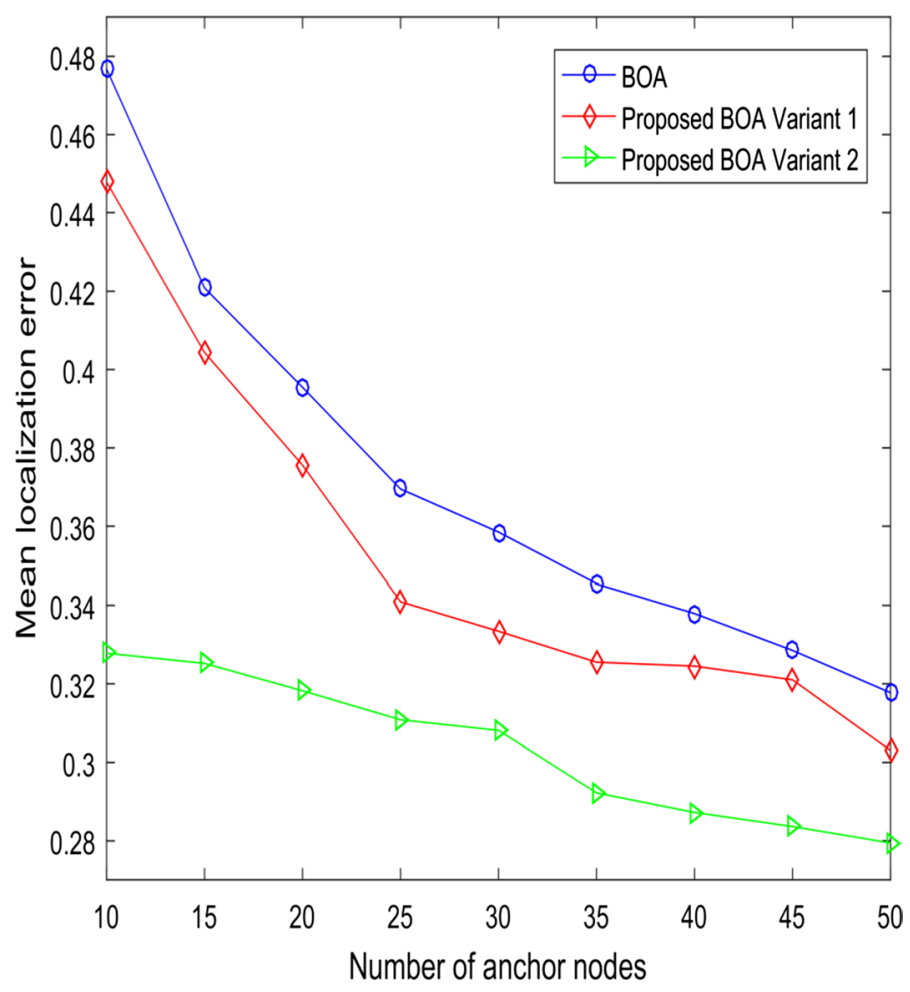

Fig. 14 Mean localization error of BOA, proposed BOA variants 1 and 2 for various numbers of anchor nodes

increased further from $100 \mathrm{~m} \times 100 \mathrm{~m}$. For $400 \mathrm{~m} \times 400 \mathrm{~m}$, the localization efficiency of the proposed BOA variant 2 is $1.5 \%$ and $52.34 \%$ higher than that of the proposed $\mathrm{BOA}$ variant 1 and $\mathrm{BOA}$, respectively, at 25 iterations.

\section{Conclusion}

The location information from where data have been obtained is required in many applications of wireless sensor networks. Therefore, the performance of WSNs depends on the localization of sensor nodes. The original bat optimization algorithm has less mean localization error and smaller computation time than other existing algorithms, but the localization efficiency is not $100 \%$ and it stuck into local optimal value. To remove these problems faced by original BOA, two variants of BOA are proposed in this paper. The proposed BOA variants 1 and 2 are modified using improved global and local search strategies in order to enhance their exploration and exploitation abilities to find the best optimum solutions. The performance of both the proposed BOA variants 1 and 2 is compared with that of other optimization algorithms such as BTOA, FA, PSO, GWO, SSA and existing BOA for various scenarios 


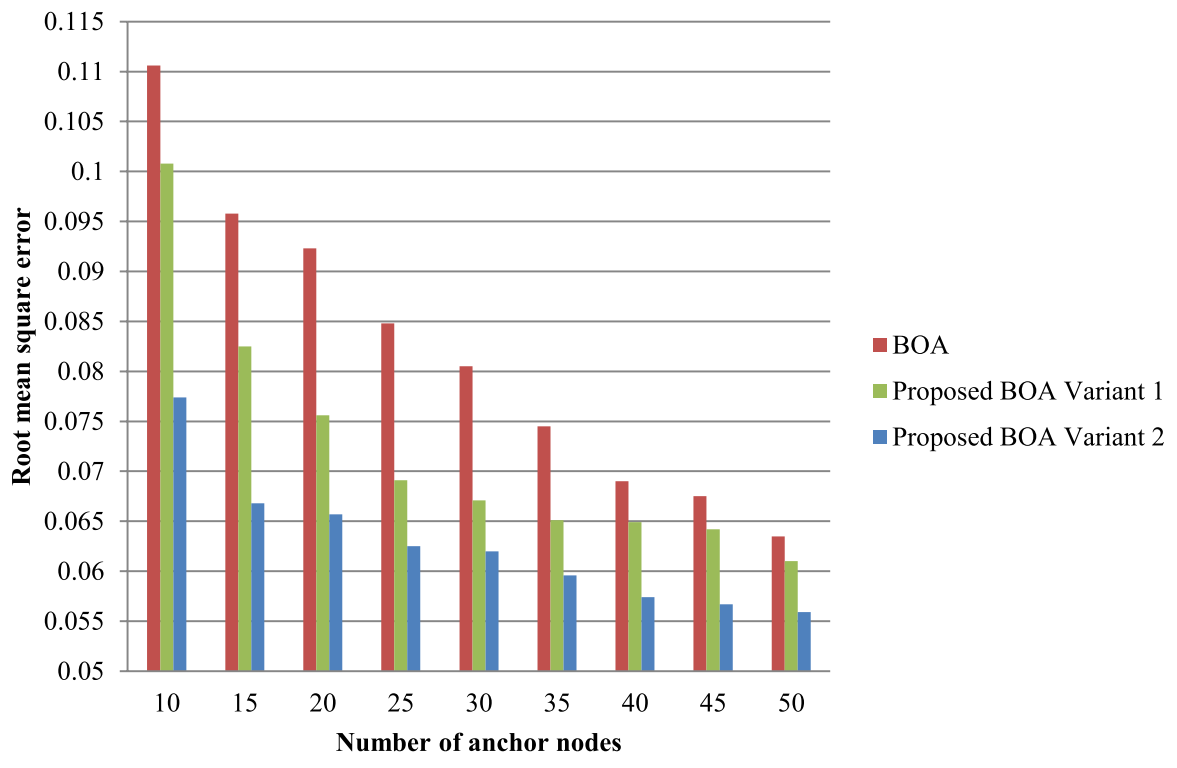

Fig. 15 Root-mean-square error of BOA, proposed BOA variants 1 and 2 for various numbers of anchor nodes

of anchor nodes and target nodes. The results revealed that the proposed BOA variant 1 and variant 2 have less mean localization error, localized more target nodes and better convergence speed, i.e. both algorithms converge at 25 iterations and take less computation time compared to other existing algorithms and original BOA. The proposed BOA variant 2 has better performance than the proposed BOA variant 1 in terms of mean localization error and speed (computation time).

Further, the comparison of BOA, the proposed BOA variants 1 and 2 in terms of various errors for several set-ups of target nodes and anchor nodes is also presented in this paper. The proposed BOA variant 2 has less average localization error, normalized localization error and root-mean-square error than those of the proposed BOA variant 1 and BOA. The proposed BOA variant 2 is more efficient than the proposed BOA variant 1 in terms of speed (less computing time) and accuracy (less value of different types of errors). Therefore, the proposed BOA variant 2 is more robust and accurate than the proposed BOA variant 1 , BOA and other existing algorithms for node localization. The limitation of the proposed BOA variant 1 is that there may be chances to include the worst solution. The drawback of the proposed BOA variant 2 is that the improved local search strategy exploits the small area around the best solution, and it does not explore the entire search space. Another demerit of the proposed BOA variant 1 is that when the size of monitoring area is increased the proposed BOA variant 1 does not localize all target nodes at 25 iterations, which reduces its localization efficiency at 25 iterations and also slows its convergence rate because 


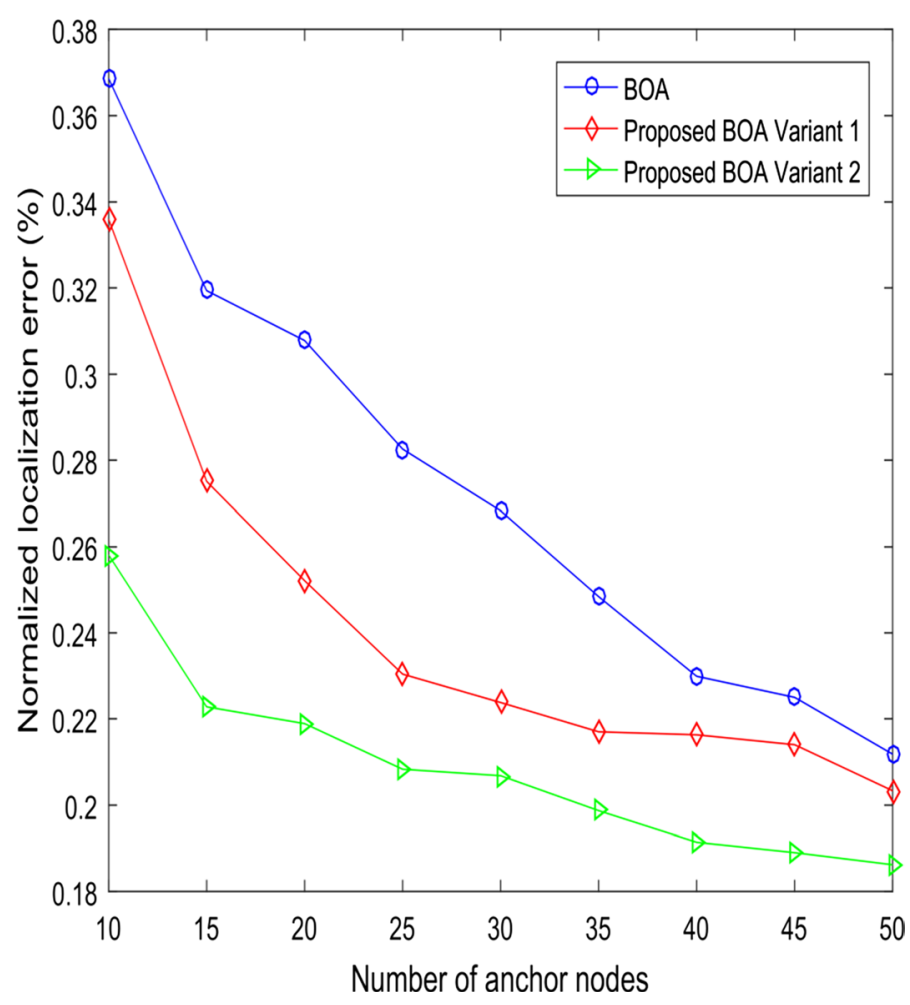

Fig. 16 Normalized localization error (\%) of BOA, proposed BOA variants 1 and 2 for various numbers of anchor nodes

Table 13 Parameters of BOA and proposed BOA variants 1 and 2 for various values of monitoring area

\begin{tabular}{lllll}
\hline Monitoring area & $\begin{array}{l}\text { Transmission } \\
\text { range } R\end{array}$ & Target nodes $N$ & Anchor nodes $M$ & $\begin{array}{l}\text { Rang- } \\
\text { ing error } \\
\text { (noise) }\end{array}$ \\
\hline $100 \mathrm{~m} \times 100 \mathrm{~m}$ & $30 \mathrm{~m}$ & 150 & 35 & $2 \%$ \\
$200 \mathrm{~m} \times 200 \mathrm{~m}$ & $60 \mathrm{~m}$ & 300 & 70 & $4 \%$ \\
$300 \mathrm{~m} \times 300 \mathrm{~m}$ & $90 \mathrm{~m}$ & 450 & 105 & $6 \%$ \\
$400 \mathrm{~m} \times 400 \mathrm{~m}$ & $120 \mathrm{~m}$ & 600 & 140 & $8 \%$ \\
\hline
\end{tabular}

the proposed BOA variant 1 converges at 50 iterations and earlier it converges at 25 iterations.

In future, a novel optimization algorithm should be proposed for localization of sensor nodes that may overcome the limitations of the proposed BOA variants 1 and 2 and localized all target nodes with more accuracy. In addition to this, the proposed BOA variants 1 and 2 can be investigated to localize the mobile sensor nodes in 
Table 14 Influence of monitoring area on performance of BOA and the proposed BOA variants 1 and 2

\begin{tabular}{|c|c|c|c|c|c|c|c|c|}
\hline \multirow[t]{2}{*}{ Monitoring area } & \multirow[t]{2}{*}{ Target nodes $N$} & \multirow[t]{2}{*}{$\begin{array}{l}\text { Number of } \\
\text { iterations }\end{array}$} & \multicolumn{2}{|c|}{ BOA } & \multicolumn{2}{|c|}{$\begin{array}{l}\text { Proposed BOA } \\
\text { variant } 1\end{array}$} & \multicolumn{2}{|c|}{$\begin{array}{l}\text { Proposed BOA } \\
\text { variant } 2\end{array}$} \\
\hline & & & $N_{L}$ & $L E(\%)$ & $N_{L}$ & $L E(\%)$ & $N_{L}$ & $L E(\%)$ \\
\hline \multirow[t]{4}{*}{$100 \mathrm{~m} \times 100 \mathrm{~m}$} & \multirow[t]{4}{*}{150} & 25 & 129 & 86.00 & 150 & 100.00 & 150 & 100.00 \\
\hline & & 50 & 143 & 95.00 & 150 & 100.00 & 150 & 100.00 \\
\hline & & 75 & 146 & 97.33 & 150 & 100.00 & 150 & 100.00 \\
\hline & & 100 & 148 & 98.66 & 150 & 100.00 & 150 & 100.00 \\
\hline \multirow[t]{4}{*}{$200 \mathrm{~m} \times 200 \mathrm{~m}$} & \multirow[t]{4}{*}{300} & 25 & 197 & 65.66 & 297 & 99.00 & 300 & 100.00 \\
\hline & & 50 & 211 & 70.33 & 300 & 100.00 & 300 & 100.00 \\
\hline & & 75 & 217 & 72.33 & 300 & 100.00 & 300 & 100.00 \\
\hline & & 100 & 231 & 77.00 & 300 & 100.00 & 300 & 100.00 \\
\hline \multirow[t]{4}{*}{$300 \mathrm{~m} \times 300 \mathrm{~m}$} & \multirow[t]{4}{*}{450} & 25 & 258 & 57.33 & 445 & 98.88 & 450 & 100.00 \\
\hline & & 50 & 261 & 58.00 & 450 & 100.00 & 450 & 100.00 \\
\hline & & 75 & 267 & 59.33 & 450 & 100.00 & 450 & 100.00 \\
\hline & & 100 & 270 & 60.00 & 450 & 100.00 & 450 & 100.00 \\
\hline \multirow[t]{4}{*}{$400 \mathrm{~m} \times 400 \mathrm{~m}$} & \multirow[t]{4}{*}{600} & 25 & 286 & 47.66 & 591 & 98.50 & 600 & 100.00 \\
\hline & & 50 & 291 & 48.50 & 600 & 100.00 & 600 & 100.00 \\
\hline & & 75 & 298 & 49.66 & 600 & 100.00 & 600 & 100.00 \\
\hline & & 100 & 305 & 50.83 & 600 & 100.00 & 600 & 100.00 \\
\hline
\end{tabular}

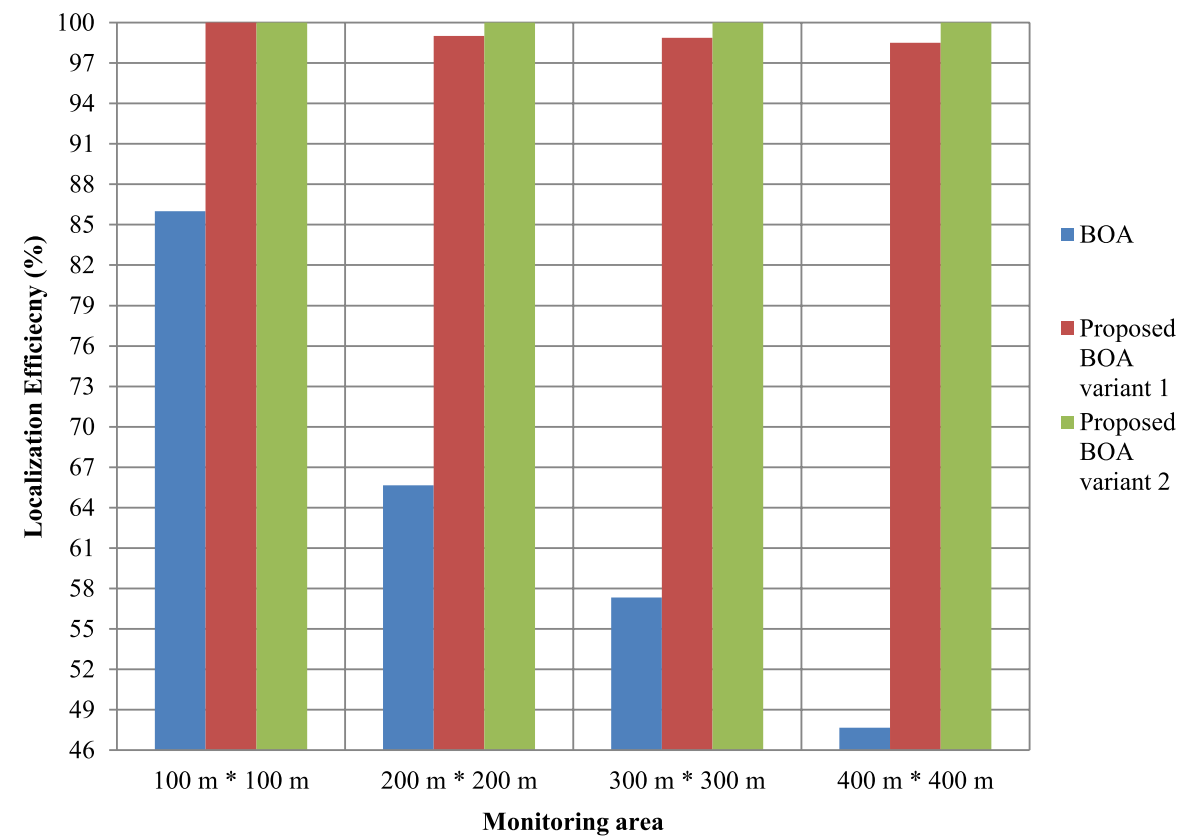

Fig. 17 Localization efficiency (\%) vs monitoring area of BOA, proposed BOA variants 1 and 2 at 25 iterations 
WSN. Moreover, hybrid evolutionary optimization algorithms can also be explored to localize the sensor nodes and to further reduce the mean localization error in twoand three-dimensional WSNs.

Acknowledgements This research is supported by the Department of Science and Technology (DST), Government of India, New Delhi, under Innovation in Science Pursuit for Inspired Research (INSPIRE) scheme Award Number IF160834.

Funding Funding was provided by the Department of Science and Technology, Ministry of Science and Technology (Grant Number DST/INSPIRE/03/2016/000251).

\section{References}

1. Aldeer MMN (2013) A summary survey on recent applications of wireless sensor networks. In: IEEE Student Conference on Research and Development (SCOReD), pp 485-490. https://doi.org/10.1109/ SCOReD.2013.7002637

2. Kulkarni RV, Kumar G, Venayagamoorthy (2011) Particle swarm optimization in wireless-sensor networks: a brief survey. IEEE Trans Syst Man Cybern Part C Appl Rev 41(2):262-267. https://doi. org/10.1109/TSMCC.2010.2054080

3. Mohar SS, Goyal S, Kaur R (2018) A survey of localization in wireless sensor network using optimization techniques. In: 4th International Conference on Computing Communication and Automation (ICCCA), pp 1-6. https://doi.org/10.1109/CCAA.2018.8777624

4. Akyildiz IF, Su W, Sankarasubramaniam Y, Cayirci E (2002) Wireless sensor networks: a survey. Comput Netw 38:393-422. https://doi.org/10.1016/S1389-1286(01)00302-4

5. Yick J, Mukherjee B, Ghosal D (2008) Wireless sensor network survey. Comput Netw 52:22922230. https://doi.org/10.1016/j.comnet.2008.04.002

6. Mohar SS, Goyal S, Kaur R (2020) Fruit fly optimization algorithm for intelligent IoT applications. In: Emerging Trends and Role of Fog, Edge and Pervasive Computing in Intelligent IoT Driven Applications, pp 284-309. https://doi.org/10.1002/9781119670087.ch16

7. Poongodi M, Hamdi M, Vijayakumar V, Rawal BS, Maode M (2020) An effective electronic waste management solution based on Blockchain Smart Contract in 5G Communities. In: IEEE 3rd 5G World Forum (5GWF), pp 1-6. https://doi.org/10.1109/5GWF49715.2020.9221346

8. Poongodi M, Hamdi M, Varadarajan V, Rawal BS, Maode M (2020) Building an authentic and ethical keyword search by applying Decentralised (Blockchain) Verification. In: IEEE INFOCOM 2020_IEEE Conference on Computer Communications Workshops (INFOCOM WKSHPS), pp 746-753. https://doi.org/10.1109/INFOCOMWKSHPS50562.2020.9162859

9. Poongodi M, Vijayakumar V, Al-Turjman F, Hamdi M, Ma M (2019) Intrusion prevention system for DDoS attack on VANET With reCAPTCHA controller using information based metrics. IEEE Access 7:158481-158491. https://doi.org/10.1109/ACCESS.2019.2945682

10. Poongodi M, Hamdi M, Sharma A, Ma M, Singh PK (2019) DDoS detection mechanism using trust-based evaluation system in VANET. IEEE Access 7:183532-183544. https://doi.org/10.1109/ ACCESS.2019.2960367

11. Poongodi M, Sundan B (2015) Stochastic model: reCAPTCHA controller based co-variance matrix analysis on frequency distribution using trust evaluation and re-eval by Aumann agreement theorem against DDoS attack in MANET. Clust Comput 18:1549-1559. https://doi.org/10.1007/ s10586-015-0496-y

12. Bizagwira H, Toussaint J, Misson M (2016) Synchronization protocol for dynamic environment: design and prototype experiments. In: 23rd International Conference on Telecommunications (ICT), pp 1-7. https://doi.org/10.1109/ICT.2016.7500369

13. Kalnoor G, Agarkhed J (2016) QoS based multipath routing for intrusion detection of sinkhole attack in wireless sensor networks. In: International Conference on Circuit, Power and Computing Technologies (ICCPCT), pp 1-6. https://doi.org/10.1109/ICCPCT.2016.7530341 
14. Khan AA, Agrawal H (2016) Optimization of delay of data delivery in Wireless Sensor Network using Genetic Algorithm. In: International Conference on Computation of Power, Energy Information and Communication (ICCPEIC), pp 159-164. https://doi.org/10.1109/ICCPEIC.2016.7557241

15. Mohar SS, Goyal S, Kaur R (2021) Optimized sensor nodes deployment in wireless sensor network using bat algorithm. Wirel Pers Commun 116(2):2835-2853. https://doi.org/10.1007/ s11277-020-07823-z

16. Singh H, Singh D (2021) Hierarchical clustering and routing protocol to ensure scalability reliability in large-scale wireless sensor networks. J Supercomput. https://doi.org/10.1007/s11227-021-03671-1

17. Singh H, Singh D (2018) Multi-level clustering protocol for load-balanced and scalable clustering in large-scale wireless sensor networks. J Supercomput 75:3712-3739. https://doi.org/10.1007/ s11227-018-2727-5

18. Singh H, Singh D (2019) An energy efficient scalable clustering protocol for dynamic wireless sensor networks. Wireless Pers Commun 109:2637-2662. https://doi.org/10.1007/s11277-019-06701-7

19. Priyadarshi R, Gupta B, Anurag A (2020) Deployment techniques in wireless sensor networks: a survey, classification, challenges, and future research issues. J Supercomput. https://doi.org/10.1007/ s11227-020-03166-5

20. Shieh CS, Sai VO, Lin YC, Lee TF, Nguyen TT, Le QD (2016) Improved node localization for WSN using heuristic optimization approaches. In: International Conference on Networking and Network Applications, pp 95-98. https://doi.org/10.1109/NaNA.2016.58

21. Zhi-kun L, Zhong L (2015) Node self-localization algorithm for wireless sensor networks based on modified particle swarm optimization. In: 27th Chinese Control and Decision Conference (CCDC), pp 5968-5971. https://doi.org/10.1109/CCDC.2015.7161879

22. Pei B, Hao Zhang H, Pei T, Wang H (2015) Firefly algorithm optimization based WSN localization algorithm. In: International Conference on Information and Communications Technologies (ICT), pp 1-5. https://doi.org/10.1049/cp.2015.0216

23. Mao G, Fidan B, Brian DOA (2007) Wireless sensor network localization techniques. Comput Netw 51:2529-2553. https://doi.org/10.1016/j.comnet.2006.11.018

24. Singha SP, Sharma SC (2015) Range free localization techniques in wireless sensor networks: a review. Procedia Comput Sci 57:7-16. https://doi.org/10.1016/j.procs.2015.07.357

25. Boukerche A, Oliveira HABF, Nakamura EF, Loureiro AAF (2007) Localization systems for wireless sensor networks. IEEE Wirel Commun 14(6):6-12. https://doi.org/10.1109/MWC.2007.4407221

26. Bertanha M, Pazzi R (2017) JLPR: joint range-based localization using trilateration and packet routing in wireless sensor networks with mobile sinks. In: IEEE Symposium on Computers and Communications (ISCC), pp 645-650. https://doi.org/10.1109/ISCC.2017.8024601

27. Singh P, Khosla A, Kumar A, Khosla M (2017) Wireless sensor networks localization and its location optimization using bio inspired localization algorithms: a survey. Int J Curr Eng Sci Res 4:74-80

28. Patwari N, Ash JN, Kyperountas S, Hero AO, Moses RL, Correal NS (2005) Locating the nodes: cooperative localization in wireless sensor networks. IEEE Signal Process Mag 22(4):54-69. https:// doi.org/10.1109/ISCC.2017.8024601

29. Halder S, Ghosal A (2015) A survey on mobility-assisted localization techniques in wireless sensor networks. J Netw Comput Appl 60:82-94. https://doi.org/10.1016/j.jnca.2015.11.019

30. Poongodi M, Hamdi M, Malviya M, Sharma A, Dhiman G, Shanmuganthan V (2021) Diagnosis and combating COVID-19 using wearable Oura smart ring with deep learning methods. Pers Ubiquitous Comput. https://doi.org/10.1007/s00779-021-01541-4

31. Poongodi M, Vijayakumar V, Rawal B, Bhardwaj V, Agarwal T, Jain A, Ramanathan L, Sriram VP (2019) Recommendation model based on trust relations \& user credibility. J Intell Fuzzy Syst 36:1-8. https://doi.org/10.3233/JIFS-169966

32. Yang XS (2013) Bat algorithm: literature review and applications. Int J Bio-Inspired Comput 5(3):110. https://doi.org/10.1504/IJBIC.2013.055093

33. Paul AK, Sato T (2017) Localization in wireless sensor networks: a survey on algorithms, measurement techniques, applications and challenges. J Sens Actuator Netw 6(4):1-23. https://doi.org/10. 3390/jsan6040024

34. Saad E, El-Hosseini M, Haikal A (2018) Recent achievements in sensor localization algorithms. Alex Eng J 57:4219-4228. https://doi.org/10.1016/j.aej.2018.11.008 
35. Gopakumar A, Jacob L (2008) Localization in wireless sensor networks using particle swarm optimization. In: IET International Conference on Wireless, Mobile and Multimedia Networks, pp 227-230. https://doi.org/10.1049/cp:20080185

36. Kulkarni RV, Venayagamoorthy GK, Cheng MX (2009) Bio-inspired node localization in wireless sensor networks. In: IEEE International Conference on Systems, Man and Cybernetics, pp 205-210. https://doi.org/10.1109/ICSMC.2009.5346107

37. Moussa A, El-Sheimy N (2010) Localization of wireless sensor network using bees optimization algorithm. In: 10th IEEE International Symposium on Signal Processing and Information Technology (ISSPIT), pp 478-481. https://doi.org/10.1109/ISSPIT.2010.5711760

38. Zhangxue Z, Huanqing C (2012) Localization in 3D sensor networks using stochastic particle swarm optimization. Wuhan Univ J Nat Sci 17(6):544-548. https://doi.org/10.1007/s11859-012-0884-6

39. Tamizharasi A, Arthi R, Murugan K (2013) Bio-inspired algorithm for optimizing the localization of wireless sensor networks. In: 4th International Conference on Computing, Communications and Network Technologies (ICCCNT), pp 1-5. https://doi.org/10.1109/ICCCNT.2013.6726485

40. Assis AF, Vieira LFM, Rodrigues MTR, Pappa GL (2013) A genetic algorithm for the minimum cost localization problem in wireless sensor networks. In: IEEE Congress on Evolutionary Computation (CEC), pp 797-804. https://doi.org/10.1109/CEC.2013.6557650

41. Goyal S, Patterh MS (2014) Wireless sensor network localization based on cuckoo search algorithm. Wirel Pers Commun 79(1):223-234. https://doi.org/10.1007/s11277-014-1850-8

42. Krishnaprabha R, Gopakumar A (2014) Performance of gravitational search algorithm in wireless sensor network localization. In: Proceeding of National Conference on Communication, Signal Processing and Networking (NCCSN), pp 1-6. https://doi.org/10.1109/NCCSN.2014.7001164

43. Zain IFM, Shin SY (2014) Distributed localization for wireless sensor networks using binary particle swarm optimization (BPSO). In: 79th Conference on Vehicular Technology (VTC Spring), pp 1-5. https://doi.org/10.1109/VTCSpring.2014.7022886

44. Li D, Wen XB (2015) An improved PSO algorithm for distributed localization in wireless sensor networks. Int J Distrib Sens Netw. https://doi.org/10.1155/2015/970272

45. Goyal S, Patterh MS (2015) Modified Bat algorithm for localization of wireless sensor network. Wirel Pers Commun 86(2):657-670. https://doi.org/10.1007/s11277-015-2950-9

46. Sai V, Shieh C, Nguyen T, Lin Y, Horng M, Le Q (2015) Parallel firefly algorithm for localization algorithm in wireless sensor network. In: 3rd International Conference on Robot, Vision and Signal Processing, pp 300-305. https://doi.org/10.1109/RVSP.2015.78

47. Peng B, Li L (2015) An improved localization algorithm based on genetic algorithm in wireless sensor networks. Cogn Neurodyn 9(2):249-256. https://doi.org/10.1007/s11571-014-9324-y

48. Goyal S, Patterh MS (2015) Flower pollination algorithm based localization of wireless sensor network. In: 2nd IEEE International Conference on Recent Advances in Engineering and Computational Sciences, pp 1-5. https://doi.org/10.1109/RAECS.2015.7453299

49. Prakash A, Yadav RK, Gupta D (2015) Sensor node deployment based on OTLBO in WSN. Procedia Comput Sci 57:998-995. https://doi.org/10.1016/j.procs.2015.07.500

50. Kumar A, Karampal (2015) Optimized range-free 3D node localization in wireless sensor networks using firefly algorithm. In: International Conference on Signal Processing and Communication, pp 14-19. https://doi.org/10.1109/ICSPCom.2015.7150611

51. Kulkarni VR, Desai V, Kulkarni RV (2016) Multistage localization in wireless sensor networks using artificial bee colony algorithm. In: IEEE Symposium Series on Computational Intelligence (SCCI), pp 1-8. https://doi.org/10.1109/SSCI.2016.7850273

52. Shayokh MA, Shin SY (2017) Bio inspired distributed WSN localization based on chicken swarm optimization. Wirel Pers Commun 97(4):5691-5706. https://doi.org/10.1007/s11277-017-4803-1

53. Rajakumar R, Amudhavel J, Dhavachelvan P, Vengattaraman T (2017) GWO-LPWSN: grey wolf optimization algorithm for node localization problem in wireless sensor networks. J Comput Netw Commun. https://doi.org/10.1155/2017/7348141

54. Arora S, Singh S (2017) Node localization in wireless sensor networks using butterfly optimization algorithm. Arab J Sci Eng 42(8):3325-3335. https://doi.org/10.1007/s13369-017-2471-9

55. Singh P, Khosla A, Kumar A, Khosla M (2017) A novel approach for localization of moving target nodes in wireless sensor networks. Int J Grid Distrib Comput 10(10):33-44. https://doi.org/10.14257/ ijgdc.2017.10.10.03

56. Strumberger I, Beko M, Tuba M, Minovic M, Bacanin N (2018) Elephant Herding Optimization Algorithm for Wireless Sensor Network Localization Problem. In: Camarinha LM, Adu KK, Julashokri M (eds) Technological Innovation for Resilient Systems Doctoral Conference on 
Computing, Electrical and Industrial Systems, IFIP Advances in Information and Communication Technology vol. 521, pp 175-184. https://doi.org/10.1007/978-3-319-78574-5_17

57. Strumberger I, Tuba E, Bacanin N, Beko M, Tuba M (2018) Monarch butterfly optimization algorithm for localization in wireless sensor networks. In: 28th International Conference Radioelektronika (RADIOELEKTRONIKA), pp 1-6. https://doi.org/10.1109/SSCI.2016.7850273

58. Ahmed MM, Houssein EH, Hassanien AE, Taha A, Hassanien E (2019) Maximizing lifetime of large-scale wireless sensor networks using multi-objective whale optimization algorithm. Telecommun Syst 72:243-259. https://doi.org/10.1007/s11235-019-00559-7

59. Gumaida BF, Luo J (2019) A hybrid particle swarm optimization with a variable neighbourhood search for the localization enhancement in wireless sensor network. Appl Intell 49:3539-3557. https://doi.org/10.1007/s10489-019-01467-8

60. Kanoosh HM, Houssein EH, Selim MM (2019) Salp swarm algorithm for node localization in wireless sensor networks. J Comput Netw Commun. https://doi.org/10.1155/2019/1028723

61. Singh P, Mittal N, Singh U, Salgotra R (2020) Naked mole-rat algorithm with improved exploration and exploitation capabilities to determine 2D and 3D coordinates of sensor nodes in WSNs. Arab J Sci Eng. https://doi.org/10.1007/s13369-020-04921-9

62. Kanwar V, Kumar A (2020) DV-Hop based localization methods for additionally deployed nodes in wireless sensor network using genetic algorithm. J Ambient Intell Humaniz Comput 11:5513-5531. https://doi.org/10.1007/s12652-020-01907-1

63. Annepu V, Rajesh A (2020) Implementation of an efficient artificial bee colony algorithm for node localization in unmanned aerial vehicle assisted wireless sensor networks. Wirel Pers Commun 114:2663-2680. https://doi.org/10.1007/s11277-020-07496-8

64. Singh P, Mittal N (2020) Efficient localisation approach for WSNs using hybrid DA-FA algorithm. IET Commun 14(12):1975-1991. https://doi.org/10.1049/iet-com.2019.1311

65. Yongyang L, Wenju L, Wang Z, Zhihao Z (2020) WSN localization technology based on hybrid GAPSO-BP algorithm for indoor three-dimensional space. Wirel Pers Commun 114:167-184. https:// doi.org/10.1007/s11277-020-07357-4

66. Qin X, Xia B, Ding T, Zhao L (2021) An improved Cuckoo search localization algorithm for UWB sensor networks. Wireless Netw 27:527-535. https://doi.org/10.1007/s11276-020-02465-2

67. Singh P, Mittal N (2021) Optimized localization of sensor nodes in 3D WSNs using modified learning enthusiasm-based teaching learning based optimization algorithm. IET Commun 15:1223-1239. https://doi.org/10.1049/cmu2.12155

68. Mohanta TK, Das DK (2021) Class topper optimization based improved localization algorithm in wireless sensor network. Wirel Pers Commun. https://doi.org/10.1007/s11277-021-08405-3

69. Ghafour MGAE, Kamel SH, Abouelseoud Y (2021) Improved DV-Hop based on Squirrel search algorithm for localization in wireless sensor networks. Wireless Netw 27:2743-2759. https://doi.org/ 10.1007/s11276-021-02618-x

70. Malathy E, Asaithambi M, Dheeraj A, Arputharaj K (2021) Hybrid bird swarm optimized quasi affine algorithm based node location in wireless sensor networks. Wireless Pers Commun. https://doi. org/10.1007/s11277-021-08934-X

71. Datta A, Dasgupta M (2021) On accurate localization of sensor nodes in underwater sensor networks: a Doppler shift and modified genetic algorithm based localization technique. Evol Intel 14:119-131. https://doi.org/10.1007/s12065-019-00343-1

72. Yang Q (2021) A new localization method based on improved particle swarm optimization for wireless sensor networks. IET Softw. https://doi.org/10.1049/sfw2.12027

73. Kanwar V, Kumar A (2021) DV-Hop-based range-free localization algorithm for wireless sensor network using runner-root optimization. J Supercomput 77:3044-3061. https://doi.org/10.1007/ s11227-020-03385-w

74. Singh P, Mittal N (2021) An efficient localization approach to locate sensor nodes in 3D wireless sensor networks using adaptive flower pollination algorithm. Wireless Netw 27:1999-2014. https://doi. org/10.1007/s11276-021-02557-7

75. Kanwar V, Kumar A (2021) DV-Hop localization methods for displaced sensor nodes in wireless sensor network using PSO. Wireless Netw 27:9-102. https://doi.org/10.1007/s11276-020-02446-5

76. Rabhi S, Semchedine F, Mbarek N (2021) An improved method for distributed localization in WSNs based on fruit fly optimization algorithm. Autom Control Comput Sci 55:287-297. https://doi.org/10. 3103/S0146411621030081 
77. Mohtavipour SM, Shahhoseini HS (2020) A link-elimination partitioning approach for application graph mapping in reconfigurable computing systems. J Supercomput 76:726-754. https://doi.org/10. 1007/s11227-019-03056-5

78. Yadav RK, Verma S, Venkatesan S (2021) iHRNL: Iterative Hessian-based manifold regularization mechanism for localization in WSN. J Supercomput 77:1-24. https://doi.org/10.1007/ s11227-021-03761-0

79. Yang XS, Gandomi AH (2012) Bat algorithm: a novel approach for global engineering optimization. Eng Comput 29(5):464-483. https://doi.org/10.1108/02644401211235834

80. Antonio C, Bestagini P, Antonacci F, Sarti A, Tubaro S (2017) Source localization and denoising: a perspective from the TDOA space. Multidimension Syst Signal Process 28:1-25. https://doi.org/10. 1007/s11045-016-0400-9

81. Whitehouse K, Karlof C, Woo A, Jiang F, Culler D (2005) The effects of ranging noise on multihop localization: an empirical study. In: 4th International Symposium on Information Processing in Sensor Networks (IPSN), pp 73-80. https://doi.org/10.1109/IPSN.2005.1440899

Publisher's Note Springer Nature remains neutral with regard to jurisdictional claims in published maps and institutional affiliations.

\section{Authors and Affiliations}

\section{Satinder Singh Mohar $^{1} \mathbb{D} \cdot$ Sonia Goyal ${ }^{1} \cdot$ Ranjit Kaur $^{1}$}

Sonia Goyal

soniagoyal@pbi.ac.in

Ranjit Kaur

ranjit24_ucoe@pbi.ac.in

1 Department of Electronics and Communication Engineering, Punjabi University Patiala, Punjab, India 